\title{
Molecular Depletion of Descending Serotonin Unmasks Its Novel Facilitatory Role in the Development of Persistent Pain
}

\author{
Feng Wei, Ronald Dubner, Shiping Zou, Ke Ren, Guang Bai, Dong Wei, and Wei Guo \\ Department of Neural and Pain Sciences, Dental School, and Program in Neuroscience, University of Maryland, Baltimore, Maryland 21201
}

Recent studies indicate that persistent pain after tissue or nerve injury is accompanied by an enhanced net descending facilitatory drive that contributes to an amplification and spread of pain. Although 5-HT-containing neurons in the rostral ventromedial medulla (RVM) provide the major descending serotonergic projection to the spinal cord, it is not clear whether the neurotransmitter 5-HT itself released from RVM-spinal neurons contributes to descending pain modulation. In the present study, we determined the role of the descending 5-HT in rat nocifensive behaviors after persistent pain by selectively depleting functional phenotypes of 5-HT in RVM neurons with regional shRNA interference (RNAi) of tryptophan hydroxylase-2 (Tph-2), the rate-limiting enzyme in the synthesis of neuronal 5-HT. Compared to negative control shRNA, Tph-2 shRNA induced significantly prolonged downregulation of Tph-2 in the RVM and 5-HT in spinal dorsal horn. The 5-HT-depleted rats showed normal pain sensitivity in responses to acute noxious stimulation. However, the same RNAi treatment attenuated formalin-induced spontaneous nocifensive responses and tissue or nerve injury-induced allodynia and hyperalgesia. Furthermore, in control shRNA-treated animals, intra-RVM microinjection of brain-derived neurotrophic factor produced a reversible hyperalgesia, which was completely prevented by Tph-2 RNAi pretreatment. Descending inhibition induced by intra-RVM electrical stimulation, but not microinjection of the $\mu$ - or $\kappa$-opioid receptor agonists in control shRNA-treated animals was eliminated in 5-HT-depleted rats. These results indicate that the descending 5-HT from the RVM is an important contributor to pain facilitation during the development of persistent pain, and may not mediate opioid-induced descending inhibition in acute pain.

\section{Introduction}

The rostral ventromedial medulla (RVM) is an important component of the descending modulatory system that constitutes a major mechanism in the control of spinal nociceptive transmission (Basbaum and Fields, 1984; Millan, 2002; Dubner and Ren, 2004). Recent studies indicate that behavioral hypersensitivity and neuronal hyperexcitability in the CNS in animal models of persistent pain are closely linked to long-lasting activation of descending modulatory circuits involving descending facilitation, which significantly contribute to the development of persistent pain after tissue and nerve injury (Porreca et al., 2002; Suzuki et al., 2004; Ren and Dubner, 2002, 2007). Despite extensive studies on the role of the RVM in descending pain modulation, the cellular and molecular mechanisms of its involvement in descending pain facilitation are not completely understood.

It has been known that there is a major spinal projection of serotonergic axons originating from an anatomically discrete group of serotonin (5-HT)-containing neurons in the brainstem, mainly in the RVM, that constitutes the descending 5-HT pathway to the spinal cord (Millan, 2002), but its role in descending pain modulation is not clear yet (Suzuki et al., 2004). Earlier studies showed that this pathway may play a role in descending pain inhibition (Basbaum and Fields, 1984). Later evidence re-

\footnotetext{
Received 0ct. 30, 2009; revised May 10, 2010; accepted May 14, 2010.

This work was supported by National Institutes of Health Grants DE18573, DE11964, NS059028, and NS060735.

Correspondence should be addressed to Dr. Feng Wei, Department of Neural and Pain Sciences, Room 8259, 650 West Baltimore Street, Baltimore, MD 21201. E-mail: fwei@umaryland.edu.

DOI:10.1523/JNEUROSCI.5389-09.2010

Copyright $\odot 2010$ the authors $\quad 0270-6474 / 10 / 308624-13 \$ 15.00 / 0$
}

veals bidirectional effects of the 5-HT system on spinal nociception (Zhuo and Gebhart, 1991; Kovelowski et al., 2000; Buhler et al., 2005). In addition, the expression of a large family of 5-HT receptor subtypes with different functions in the dorsal horn may mediate opposing effects on the descending 5-HT system in spinal pain processing (Hamon and Bourgoin, 1999; Suzuki et al., 2004; Lopez-Garcia, 2006; Dogrul et al., 2009), making it difficult to identify their role in enhancing descending pain modulation during chronic pain states.

Previous studies showed that selective lesions or lack of 5-HTcontaining neurons in the RVM or the hindbrain by focal neurotoxin treatment (Wei et al., 1999a) or genomic disruption in $L m \times 1 b^{\mathrm{f} / \mathrm{f} / \mathrm{p}}$ mice (Zhao et al., 2007), respectively, cause enhanced inflammatory pain, suggesting that active 5-HT-containing neurons in the RVM had an elevated descending inhibitory influence during persistent pain states. It is important to note that multiple neurotransmitter systems have been found to coexist and several functional receptors express in RVM 5-HT-containing neurons (Hökfelt et al., 2000; Millan, 2002). Thus, it is not clear whether the enhanced nociception after deletion of RVM 5-HT-containing neurons is due to the loss of 5-HT action at spinal sites or to the loss of other coexisting inhibitory neurotransmitters, or even to the loss of the integrated effects from multiple receptors expressed in 5-HT-containing neurons. To determine a more definitive role of the descending 5-HT in spinal pain processing, we generated rats with the selective loss of functional phenotypes of 5-HT in RVM neurons with regional shRNA interference (RNAi) of neuronal tryptophan hydroxylase-2 (Tph-2), the ratelimiting enzyme in the synthesis of 5-HT in the CNS (Invernizzi, 
2007), and assessed the contribution of the RVM 5-HT system to descending pain modulation by examining nocifensive behavior in different persistent pain models.

\section{Materials and Methods}

Animals. Adult male Sprague Dawley rats weighing 175-350 g (Harlan) were used in all experiments. Rats were on a $12 \mathrm{~h}$ light/dark cycle and received food and water ad libitum. The experiments were approved by the Institutional Animal Care and Use Committee of the University of Maryland Dental School.

Intra-RVM microinjections and targeted recombinant plasmid transfers by electroporation. As previously described (Guo et al., 2006), rats were anesthetized with 2-3\% isoflurane in a gas mixture of $30 \% \mathrm{O}_{2}$ balanced with nitrogen and placed in a Kopf stereotaxic instrument (Kopf Instruments). A midline incision was made after infiltration of lidocaine (2\%) into the skin. A midline opening was made in the skull with a dental drill to insert a microinjection needle into the target site. The RVM is termed for collective structures that consist of the midline nucleus raphe magnus (NRM) and the adjacent gigantocellular reticular nucleus $\alpha$ part $(\mathrm{NGC} \alpha)$. The coordinates for the NRM were as follows: $10.5 \mathrm{~mm}$ caudal to bregma, midline, and $9.0 \mathrm{~mm}$ ventral to the surface of the cerebellum (Paxinos and Watson, 2005). To avoid penetration of the transverse sinus, the incisor bar was set at $4.7 \mathrm{~mm}$ below the horizontal plane passing through the interaural line. Animals were subsequently maintained at $\sim 1 \%$ halothane. Microinjections were performed by delivering drug solutions slowly over a $10 \mathrm{~min}$ period using a $0.5 \mu \mathrm{l}$ Hamilton syringe with a 32 gauge needle. For gene transfer, SureSilencing shRNA plasmids for Rat Tph-2 were used to design the enclosed shRNA (Tph-2: TCAACATGCTCCATATTGAAT) or scrambled control (negative control Tph-2: ggaatctcattcgatgcatac) and contained the GFP gene (SuperArray). Each vector $(0.5 \mu \mathrm{g} / 0.5 \mu \mathrm{l})$ was injected into the RVM. The injection needle was left in place for at least $15 \mathrm{~min}$ before being slowly withdrawn. A pair of Teflon-coated silver positive and negative electrodes were placed around the microinjection sites rostrocaudally. For transfer of negatively charged plasmid into RVM neurons, seven square-wave electric pulses (50 ms, $40 \mathrm{~V}, 1 \mathrm{~Hz}$; model 2100; A-M Systems) were delivered. The wound was closed and animals returned to their cages after they recovered from anesthesia. In some experiments, control or Tph-2 shRNA plasmids were injected to the RVM and then followed by placing electrodes without electroporation. In addition, some groups of animals at $3 \mathrm{~d}$ after gene transfer were subjected to injection of the human recombinant brain-derived neurotrophic factor (BDNF, $100 \mathrm{fmol}$, Amgen) (Guo et al., 2006), the $\mu$-opioid receptor agonist [D-Ala ${ }^{2}, \mathrm{NMePhe}^{4}$, Gly-ol $^{5}$ ] enkephalin (DAMGO, 40 or $100 \mathrm{pmol} / 0.5 \mu \mathrm{l}$, Sigma) (Hurley et al., 2003), or the $\kappa$-opioid receptor (KOR) agonist trans-( \pm )-3,4dichloro- $N$-methyl- $N$-[2-(1-pyrrolidinyl)cyclohexyl] benzeneacetamide hydrochloride (U50488, 5 or $20 \mathrm{nmol} / 0.5 \mu \mathrm{l}$, Sigma) (Hirata et al., 2000) or $N$-methyl- $N$-[(5R,7S,8S)-7-(1-pyrrolidinyl)-1-oxaspiro(4.5)dec-8yl]benzeneacetamide (U69593, 0.1, 0.5, or $3 \mathrm{nmol} / 0.5 \mu \mathrm{l}$ ) (Pan et al., 1997; Ackley et al., 2001). BDNF, DAMGO, and U50488 were dissolved in ACSF. U69593 was dissolved in 10\% (w/v) 2-hydroxy-b-cyclodextrin, Sigma). The control rats underwent identical procedures with injection of the same volume $(0.5 \mu \mathrm{l})$ of the vehicles. All wound margins were covered with a local anesthetic ointment (Nupercainal; Rugby Laboratories), the wound was closed, and animals were returned to their cages after they recovered from anesthesia.

Pain models and behavioral tests. Animals were placed in clear plastic chambers on an elevated table and allowed to acclimate for $\sim 30 \mathrm{~min}$. Nociceptive responses to thermal and mechanical stimuli were measured as described previously (Hargreaves et al., 1988; Guo et al., 2006). To examine whether there were effects of descending 5-HT depletion and blocking of synthesis on the function of spinal ventral horn neurons, motor function was performed on an accelerating rotarod treadmill as described preciously (Wei et al., 1999b). For the formalin test, the total time spent licking and scratching the injected hindpaw was recorded during each $5 \mathrm{~min}$ interval for $2 \mathrm{~h}$ after intraplantar injection of $2 \%$ formalin $(50 \mu \mathrm{l})$. For an inflammatory pain model, complete Freund's adjuvant (CFA, $50 \mu \mathrm{l}$, containing $25 \mu \mathrm{g}$ of Mycobacterium tuberculosis) was injected subcutaneously into the plantar surface of the left hindpaw (Wei et al., 1999a). To establish a persistent pain model with spinal nerve ligation (SNL), under anesthesia with 3\% isoflurane, the left L5 spinal nerve was exposed and tightly ligated with 4-0 soft silk thread. Sham surgery was used as a control. The paw withdrawal latency (PWL) to radiant heat was measured before and after gene transfer, hindpaw inflammation, or SNL. The mechanical sensitivity was measured with a series of calibrated von Frey filaments before and after gene transfer and tissue or nerve injury. An $\mathrm{EF}_{50}$ value was defined as the von Frey filament force (in grams) that produced a 50\% frequency of the paw withdrawal responses and was used as a measure of mechanical sensitivity (Guo et al., 2004). Body weight and hindpaw diameters were determined before and after gene transfer as well as at 1 and $3 \mathrm{~d}$ after inflammation. All behavioral tests were conducted under blind conditions.

Intra-RVM electrical stimulation. Rats were weakly anesthetized with isoflurane and mounted in a stereotaxic apparatus. The stimulation site in the RVM was located stereotaxically as described above. A monopolar electrode consisting of Teflon-insulated stainless steel wire $(0.1 \mathrm{~mm})$ exposed only at the cut tip was introduced. The indifferent electrode was attached to the neck muscles. The RVM was stimulated with monopolar square-wave pulses $(0.5 \mathrm{~ms}, 50 \mathrm{~Hz}, 100 \mu \mathrm{A})$ for $20 \mathrm{~s}$ as described by Hammond and Yaksh (1984). Before, during, and at 5, 10, and $15 \mathrm{~min}$ after electrical stimulation (ES), the paw withdrawal latency to radiant heat was measured. The sham group received an electrode placement without ES treatment and did not show any differences in the PWL before and after electrode was placed.

Immunohistochemistry. At different time points after gene transfer, rats were deeply anesthetized with $3 \%$ isoflurane and perfused transcardially with $200 \mathrm{ml}$ of saline followed by $500 \mathrm{ml}$ of cold $\left(4^{\circ} \mathrm{C}\right) 0.1 \mathrm{M}$ phosphate buffer (PB) containing $4 \%$ paraformaldehyde. The forebrain, brainstem, and lumbar spinal cord (at L4-6) was removed, immersed in the same fixative overnight at $4{ }^{\circ} \mathrm{C}$, and transferred to $30 \%$ sucrose $(\mathrm{w} / \mathrm{v})$ in phosphate buffer for several days for cryoprotection. Thirty-micrometerthick coronal sections of the brainstem were cut with a cryostat at $-20^{\circ} \mathrm{C}$. To determine effects of Tph-2 shRNA or scrambled shRNA on expression of Tph-2, or 5-HT in neurons transferred with GFP, free-floating tissue sections were incubated with rabbit anti-Tph-2 (1:2000, Millipore) and anti-5-HT (1:5000, ImmunoStar) antibody overnight. After washes, the sections were incubated with AffiniPure biotinylated secondary IgG (1:800, Jackson ImmunoResearch Laboratories) for $1 \mathrm{~h}$. StreptavidinAlexa 568 or 674 (1:800, Invitrogen) was used for fluorescence staining of Tph-2 or 5-HT. Control staining procedures were performed by omission of the primary or secondary antibodies, and by preabsorption with an excess $(10 \mu \mathrm{g} / \mathrm{ml})$ of the respective antigens. To examine colocalization of 5 -HT with Tph-2 or $\mu$-opioid receptor (MOR) within RVM neurons, double labeling for both 5-HT and Tph-2 or MOR in the RVM of naive rats $(n=3)$ without gene transfer was performed simultaneously with rabbit anti-Tph- 2 or anti-MOR (1:8000, Incstar) and goat anti5-HT (1:500, Millipore). The sections then were incubated for $2 \mathrm{~h}$ in solutions containing species-specific secondary antibodies coupled to Alexa 568 or 488 , respectively. In addition, to identify spinally projecting RVM neurons, a series of microinjections (4-6 sites) of a retrograde tracer Fluoro-Gold (FG, $50 \mathrm{nl}, 1 \%$ in saline, Fluorochrome) were made in the bilateral lumbar spinal dorsal horn (L4-5). Animals were perfused $6 \mathrm{~d}$ after injections. Tyramide signal amplification was used for triple immunostaining as described previously (Guo et al., 2006). RVM tissue sections were processed for retrograde FG, TrkB, and 5-HT immunoreactivity by incubation in rabbit anti-FG (1:5000, Millipore Bioscience Research Reagents), anti-TrkB (1:1000, Millipore Bioscience Research Reagents), and goat anti-5-HT individually. After incubation in biotinylated secondary antibody and Streptavidin-conjugated peroxidase, three Alexa Fluor $(488,568$, or 637$)$-conjugated tyramides were used to visualize labeling, respectively. After washes, all sections were mounted on gelatin-coated slides and coverslipped with Vectashield (Vector Laboratories). Images were collected sequentially using a Zeiss 510 MATA laser-scanning confocal microscope with Axiovert 2000 system. Adobe Photoshop (version CS) was used for image cropping and adjustment. In addition, estimates of 5-HT-containing neurons in the RVM, including NRM and the adjacent NGC $\alpha$, were obtained from four rats in each 
group processed for 5-HT immunohistochemistry and DAPI counterstaining (Fluoro-Gel II, Electron Microscopy Sciences). Ten sections from a 1:5 series were analyzed for each brainstem. Neurons were identified and counted only if their nuclei were fully stained with DAPI and surrounded by 5 -HT-labeled cytoplasm and proximal dendrites. The investigator responsible for plotting and counting the labeled cells was blind to the experimental situation of each animal.

Western blot. Naive and treated rats were anesthetized with $2 \%$ halothane and decapitated. The RVM tissues were removed as previously described (Guo et al., 2006) and homogenized in solubilization buffer. The homogenate was centrifuged at 20,200 $\times g$ for $10 \mathrm{~min}$ at $4^{\circ} \mathrm{C}$, and the supernatant was removed. The protein concentration was determined. Each sample contained proteins from one animal. The proteins $(50 \mu \mathrm{g})$ were separated on a 7.5\% SDS-PAGE gel and blotted to a nitrocellulose membrane (GE Healthcare). The blot was incubated with rabbit antiTph-2 antibody overnight at $4^{\circ} \mathrm{C}$. The membrane was washed with TBS and incubated for $1 \mathrm{~h}$ with anti-goat IgG horseradish peroxidase (HRP) (1:3000; Santa Cruz Biotechnology) in 5\% milk/TBS. The immunoreactivity was detected using enhanced chemiluminescence (ECL) (GE Healthcare). The loading and blotting of equal amount of proteins were verified by reprobing the membrane with anti- $\beta$-actin antiserum (Sigma). The ECL-exposed films were digitized, and densitometric quantification of immunoreactive bands was performed using U-SCAN-IT gel (version 4.3, Silk Scientific).

5-HT ELISA. Rat serotonin ELISA kit was purchased from ALPCO Diagnostics. Animals were anesthetized with $2 \%$ halothane and decapitated at $1,3,5,7$, and $10 \mathrm{~d}$ after Tph-2 shRNA or control plasmid gene transfer. The dorsal part of lumbar spinal cord segments was dissected. Spinal cord tissues were homogenized in a lysis buffer containing protease and phosphatase inhibitors. The concentrations of protein were measured by BCA Protein Assay (Pierce). For each reaction in a 96-well plate, $12 \mu \mathrm{g}$ of proteins were used, and ELISA was performed according to the protocol of the manufacturer. The standard curve was included in each experiment.

Histological reconstruction. The location of microinjection and identification of successful gene transfer in the RVM were determined by visualization of GFP expression. Rats with misplaced microinjection or GFP expression were excluded from the data analysis. The location of electrical stimulation in the RVM was examined after the animals were deeply anesthetized with isoflurane and perfused with fixative, and the RVM tissue was dissected.

Data analysis. Results were expressed as mean \pm SEM. Statistical comparisons included Student's $t$ test or one- or two-way ANOVA with the post hoc Scheffe's $F$ test in Western blot analysis and cell counting or the Student-Newman-Keuls test in behavioral experiments (ANOVA with repeated measures). In all cases, $p<0.05$ was considered statistically significant.

\section{Results}

Normal distribution of 5-HT neurons in the RVM and 5-HT fibers in spinal dorsal horn

Our previous study demonstrated a normal distribution of 5-HT-containing neurons in rat RVM, including the NRM and the adjacent NGC $\alpha$, and 5-HT fibers in the spinal dorsal horn (Wei et al., 1999a). Recently, a new isoform of tryptophan hydroxylase, named tryptophan hydroxylase-2 (Tph-2), that catalyzes the transformation of tryptophan into $5-\mathrm{HT}$, was found in brain as the rate-limiting enzyme in neuronal serotonin biosynthesis and can be used as a marker of central 5-HT-containing neurons (Walther et al., 2003; Zhang et al., 2004; Invernizzi, 2007). Although immunoreactivity of broad Tph (both central Tph-2 and peripheral Tph-1) was formerly used for identification of RVM 5-HT neurons (Wang and Wessendorf, 1999; Marinelli et al., 2002; Close et al., 2009), the normal expression of Tph-2 and its anatomical relationship with 5-HT in the RVM had been not reported. Therefore, we first examined the distribution of Tph-2 in the RVM by immunostaining. At low magnification,

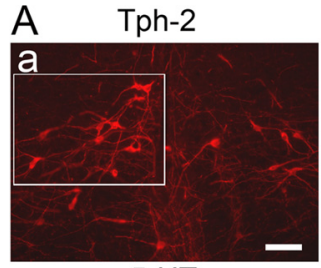

$5-\mathrm{HT}$

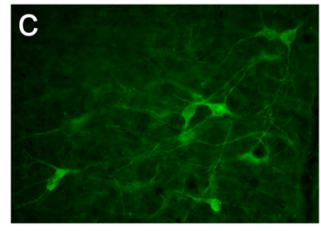

B $\quad 5-\mathrm{HT}$

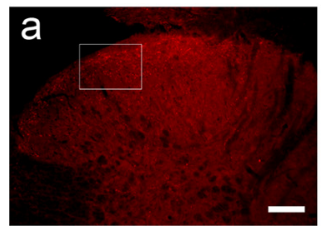

Tph-2

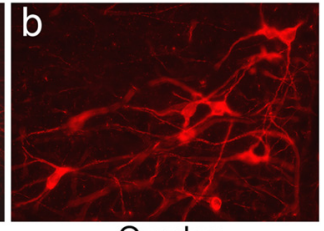

Overlap

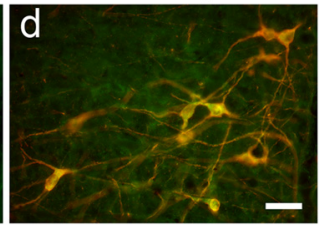

$5-\mathrm{HT}$

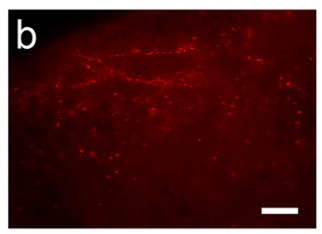

Figure 1. Immunostaining shows the colocalization of Tph-2 with 5-HT in neurons of the RVM $(\boldsymbol{A})$ and the distribution of 5 - $\mathrm{HT}$ fibers in the spinal dorsal horn $(\boldsymbol{B})$. $\boldsymbol{A a}$, Normal distribution of Tph-2-labeled neurons in low power of a tissue section at the rostral medulla level of the naive rats $(n=3)$. $\boldsymbol{A} \boldsymbol{b}$ is enlarged from the inset $(\boldsymbol{A a})$, illustrating the distribution of Tph-2immunoreactive neurons in the RVM. $\boldsymbol{A c}, 5-\mathrm{HT}$ immunostaining in RVM neurons shown in $\boldsymbol{A} \boldsymbol{b}$. $\boldsymbol{A d}$, Expression of Tph-2 with 5-HT in the same RVM neurons. Ba, 5-HT-labeled fibers and terminals in spinal dorsal horn of the naive rats $(n=3)$ at $L 5$ level. $\boldsymbol{B} \boldsymbol{b}$, An enlarged image of 5 -HT fibers in the superficial dorsal horn cord from the insets (Ba). Scale bars: $100 \mu \mathrm{m}(\boldsymbol{A a}, \boldsymbol{A b}$, Ba), $50 \mu \mathrm{m}(\boldsymbol{A c}, \boldsymbol{A d}), 20 \mu \mathrm{m}(\boldsymbol{B b})$.

there is a typical expression of Tph-2-labeled neurons with mediolateral extending dendrites (Fig. $1 \mathrm{Aa}$ ). In addition, coimmunostaining with 5-HT revealed a complete colocalization of both markers, as shown at higher magnification (Fig. $1 A b-A d$ ). Spinal 5-HT expression was also examined in the spinal dorsal horn from L4-6 levels. Immunostaining demonstrated that many varicosity-like 5-HT fibers or terminals were mainly expressed in superficial dorsal horn (Fig. $1 B$ ), lamina $\mathrm{X}$, and ventral horn (data not shown).

\section{Selective knockdown of Tph-2 expression in the RVM by Tph-2 RNAi}

To investigate whether the RVM 5-HT system was involved in descending modulation of spinal pain transmission, we selectively knocked down endogenous Tph-2 expression by transferring a plasmid encoding Tph-2 shRNA into the RVM to induce Tph-2 silencing and decrease functional 5-HT synthesis by taking advantage of the electroporation technique in vivo (Wei et al., 2003; Guo et al., 2006). A single injection of plasmid (500 ng) for Tph-2 shRNA or scrambled (control) shRNA was microinjected into the RVM of the adult rats and followed by local electroporation. To verify the efficiency of plasmid transfer, the GFP gene was encoded in both plasmids and GFP expression in the RVM cells after transfer was examined under the fluorescence microscope. We observed a wide GFP expression in cells limited to the RVM area, mainly the NRM, from $1 \mathrm{~d}$ to $7 \mathrm{~d}$ after gene transfer (Fig. 2A, B), indicating a successful transfection of the plasmids in RVM neurons identified by GFP expression after microinjection and following electroporation. In the rats receiving control shRNA, intense Tph-2 immunoreactivity was still monitored in RVM neurons in which GFP expression was detected for up to $7 \mathrm{~d}$ after gene transfer. As shown in Figure $2 \mathrm{~A}$, 


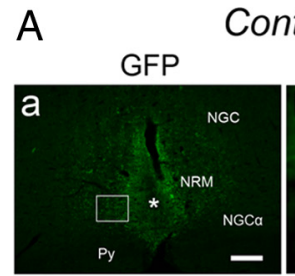

Control
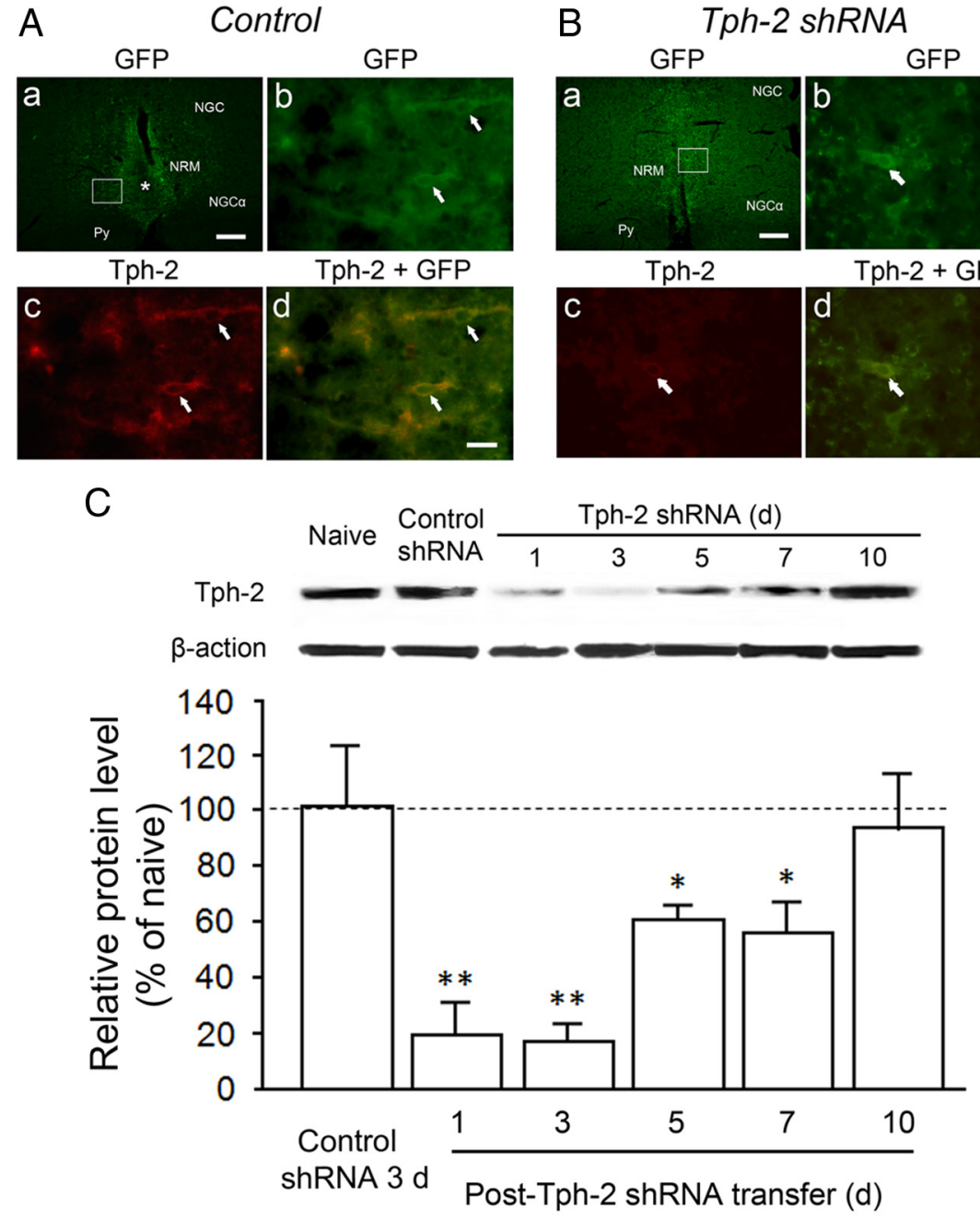

$\mathrm{D}$

DRN

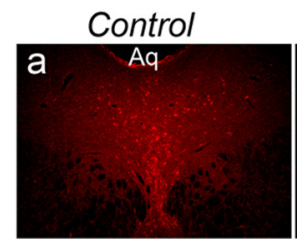
T/jh-2 ShRNA
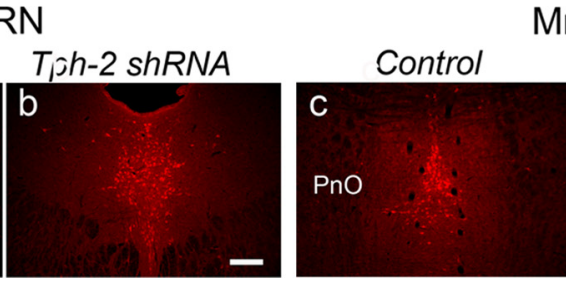

$\mathrm{MnR}$

Figure 2. Expression of GFP and Tph-2 immunostaining in RVM neurons after electroporation transfer of plasmids and RNAi with Tph-2 shRNA or control shRNA. A, GFP expression in the RVM neurons (arrows, $\boldsymbol{A} \boldsymbol{b}$ ) at $3 \mathrm{~d}$ after control gene transfer is magnified from the inset in $\boldsymbol{A a}$. The asterisk $(\boldsymbol{A} \boldsymbol{a})$ indicates the track of microinjection needle. Normal Tph-2 immunoreactivity $(\boldsymbol{A c})$ is present in GFP-expressing neurons ( $\boldsymbol{A} \boldsymbol{d}$ ). $\boldsymbol{B}$, GFP expression in the RVM neurons (arrows, $\boldsymbol{B} \boldsymbol{b}$ ) at $3 \mathrm{~d}$ after treatment with Tph-2 shRNA is enlarged from the inset in $\boldsymbol{B} \boldsymbol{a}$. However, compared with the immunostaining in $\boldsymbol{A c}$, significant decrease or loss of Tph-2 immunoreactivity in the RVM neurons $(\boldsymbol{B C})$ is shown in the GFP-expressing cells (arrow, $\boldsymbol{B} \boldsymbol{b}-\boldsymbol{B} \boldsymbol{d}$ ). $\boldsymbol{C}$, Western blot illustrating long-lasting decrease or depletion in Tph-2 in the RVM tissues from $1 \mathrm{~d}$ to $7 \mathrm{~d}$ after gene transfer $\left[n=3,{ }^{*} p<0.05\right.$; ${ }^{* *} p<0.01$, vs control shRNA $(n=3)]$. $\boldsymbol{D}, 5-\mathrm{HT}$ immunostaining in the neighboring serotonergic nuclei, including the DRN $(\boldsymbol{D a}, \boldsymbol{D b})$ and the median raphe nucleus $(\mathrm{MnR}, \boldsymbol{D} \boldsymbol{c}, \boldsymbol{D d})$, at $4 \mathrm{~d}$ after control $(\boldsymbol{D a}, \boldsymbol{D c})$ or Tph-2 shRNA (Db, $\boldsymbol{D d})$ treatment in the RVM. Scale bars: 100 $\mu \mathrm{m}(\boldsymbol{A a}, \boldsymbol{B} \boldsymbol{a}, \boldsymbol{D a}-\boldsymbol{D d}) ; 30 \mu \mathrm{m}(\boldsymbol{A b}-\boldsymbol{A d}, \boldsymbol{B} \boldsymbol{b}-\boldsymbol{B d})$. Aq, Aqueduct; Pn0, pontine reticular nucleus, oral part; Py, pyramidal tract.

Tph-2 expression at $3 \mathrm{~d}$ after control gene transfer was similar to that in naive rats (see Fig. $1 A$ ). In contrast, immunostaining showed no or less Tph-2 immunoreactivity in GFP-expressing neurons in the RVM at $3 \mathrm{~d}$ after transferring the plasmid of Tph-2 shRNA (Fig. $2 B$ ), whereas strong MOR immunostaining was still observed in GFP-expressing neurons in the RVM and untransfected neurons in nearby nuclei (data not shown). To quantitatively assess the effects of Tph-2 RNAi on Tph-2 expression in the RVM, Western blots were performed to measure the time course

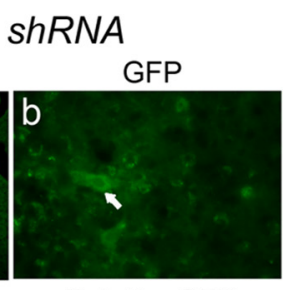

Tph-2 + GFP

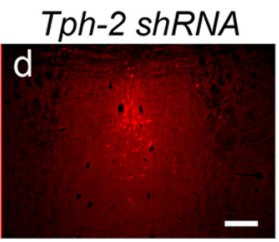

changes of Tph-2 protein levels in RVM tissue for periods up to $10 \mathrm{~d}$ after gene transfer. When tested at $3 \mathrm{~d}$ after gene transfer, control shRNA ( $n=5$ rats) did not reduce the Tph-2 level when compared to naive animals $(101.3 \pm 23.0 \%$ of naive level $n=6, p>0.05$ ) (Fig. $2 C$ ). The same treatment was also ineffective for deletion of Tph-2 level in the RVM at other time points, including $1 \mathrm{~d}$ and $5-10 \mathrm{~d}(n=$ 3 for each time point, $p>0.05$ vs naive, data not shown). However, the Tph-2 shRNA induced a remarkable downregulation of Tph-2 protein of nearly $80 \%$ at $1-3 \mathrm{~d}(19.3 \pm 11.9 \%$ at $1 \mathrm{~d}$ and $16.3 \pm$ $6.3 \%$ at $3 \mathrm{~d}, p<0.01$ vs control shRNA, $n=6$ for each time point) (Fig. 2C) and $\sim 50 \%$ at 5 and $7 \mathrm{~d}(60.4 \pm 5.2 \%$ at $5 \mathrm{~d}$, $55.3 \pm 11.8 \%$ at $7 \mathrm{~d}, p<0.05$ vs control, $n=3$ ) after gene transfer. The Tph-2 levels in the RVM at $10 \mathrm{~d}$ after plasmid injection returned to control levels $(p>0.05$ vs control, $n=3$ for each). We also examined the possible effects of Tph-2 shRNA in the RVM on Tph-2 expression in more rostral brainstem 5-HT nuclei, including the dorsal raphe nucleus (DRN) and the median raphe nucleus, in which immunostaining showed similar 5-HT expression when compared to that from the control shRNA-treated group (Fig. 2D). These results suggest a significant and selective deletion or downregulation of Tph-2 in RVM 5-HT neurons following RNAi.

\section{Prolonged and effective depletion of 5-HT in the RVM and spinal dorsal horn after Tph-2 RNAi}

Because Tph-2 shRNA was most effective in reducing Tph-2 expression up for 1 week, we then examined the consequence of Tph-2 downregulation on 5-HT synthesis in the RVM and its expression in spinal cord in a series of parallel experiments. We found 5-HT immunoreactivity present in GFP-expressing neurons at $3 \mathrm{~d}$ after control gene injection $(n=5)$ (Fig. $3 A)$, whereas treatment with Tph-2 shRNA prevented 5-HT expression in transferred RVM neurons, the majority being neurons in NRM identified by GFP expression $(n=5)$ (Fig. 3B). Consistent with the Western blot data for Tph-2 change, the mean number of 5 -HTimmunolabeled neurons per section in the RVM was significantly lower by $75 \%$ in the 5 -HT-depleted rats $(9.65 \pm 4.57, p<0.01$, $n=4)$ compared with the control shRNA groups $(37.11 \pm 8.83$, $n=4)$ at $3 \mathrm{~d}$ after gene transfer. Furthermore, the numbers of 5 -HT-labeled neurons were reduced by $94 \%$ in the NRM and $46 \%$ in the NGC $\alpha$ from the Tph-2 shRNA-treated group when compared to that in control rats (Fig. $3 B, C$ ). These data suggest that the 5-HT expression in the NRM was almost completely depleted, and the remaining 5-HT-labeled neurons commonly 
existed in the NGC $\alpha$, which is distant from the microinjection site and genetransferred areas. No significant differences were found between naive ( $41.82 \pm$ 6.28, $n=4$ ) and control shRNA-treated animals for 5-HT-immunostaining neurons counted in the RVM $(p>0.05)$. Moreover, reduction of the number and density of 5-HT-labeled varicosity-like processes was observed in the bilateral spinal dorsal horns, especially in the superficial dorsal horn at $3 \mathrm{~d}$ after transfer of the plasmid of Tph-2 shRNA $(n=5)$ (Fig. $3 D)$, whereas control shRNA did not affect 5-HT expression in the same area $(n=5)$ (Fig. 3D). The 5-HT expression in the ventral horn was not affected in rats with treatment of Tph-2 RNAi (data not shown). We further quantitatively examined the time course changes of tissue 5-HT levels in the spinal dorsal horn at the lumber enlargement at different time points after gene transfer in the RVM by ELISA. As shown in Figure 3E, there was no significant change in mean concentrations of 5-HT at $3 \mathrm{~d}$ after control gene transfer in comparison to naive level $[n=$ 4 for each groups, $6.65 \pm 0.57$ vs $7.36 \pm$ 0.57 (ng/mg spinal cord protein), respectively, $p>0.05$ ] (Fig. 3E). After Tph-2 shRNA gene transfer, the levels of 5-HT in the spinal dorsal horn were significantly decreased at $1 \mathrm{~d}(n=3,4.17 \pm 0.86 \mathrm{ng} /$ mg, $p<0.05$ vs control shRNA), $3 \mathrm{~d}$ ( $n=$ $3,2.2 \pm 0.12 \mathrm{ng} / \mathrm{mg}, p<0.01$ vs control), $5 \mathrm{~d}(n=3,2.93 \pm 0.10 \mathrm{ng} / \mathrm{mg}, p<0.05$ vs control), and $7 \mathrm{~d}(n=3,4.12 \pm 0.97 \mathrm{ng} /$ $\mathrm{mg}, p<0.05$ vs control), and it returned to the naive level at $10 \mathrm{~d}(n=3,5.68 \pm$ $1.21 \mathrm{ng} / \mathrm{mg}$ ) after gene transfer (Fig. $3 E$ ). Together, these results demonstrate that in contrast with rats treated with the scrambled gene, the levels of 5-HT in the descending 5-HT system, mainly NRM neurons, and their terminals in the spinal dorsal horn, showed a prolonged and effective depletion for up to 5-7 d, peaking at $3 \mathrm{~d}$ after a single transfer of the plasmid encoding Tph-2 shRNA, although residual 5-HT remained in the NGC $\alpha$ and spinal dorsal horn.

\section{Normal nociception and mobility after the intra-RVM 5-HT depletion}

To investigate the functional consequence of RVM 5-HT deletion, we first compared control (scrambled shRNA-treated) and RVM 5-HT-depleted (Tph-2 shRNA-treated) rats in response to thermal and mechanical measures of acute pain (Fig. $4 A, B$, respectively) for $7 \mathrm{~d}$ in which there was significant downregulation of 5-HT in both the RVM and the spinal dorsal horn tissue. There was no significant difference in thermal withdrawal latency and mechanical withdrawal threshold $\left(\mathrm{EF}_{50}\right)$ between control $(n=8$ rats) and the RVM 5-HT-deleted group $(n=8)$ in baseline before
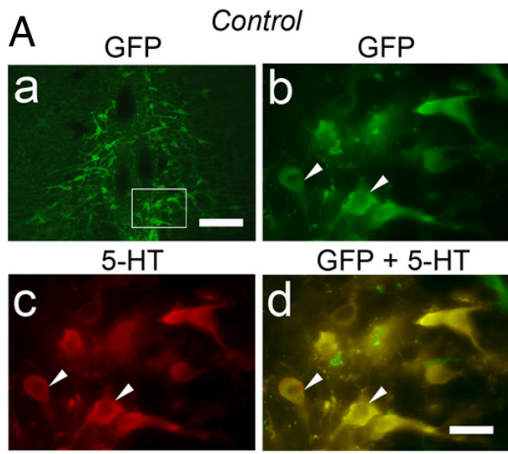

C
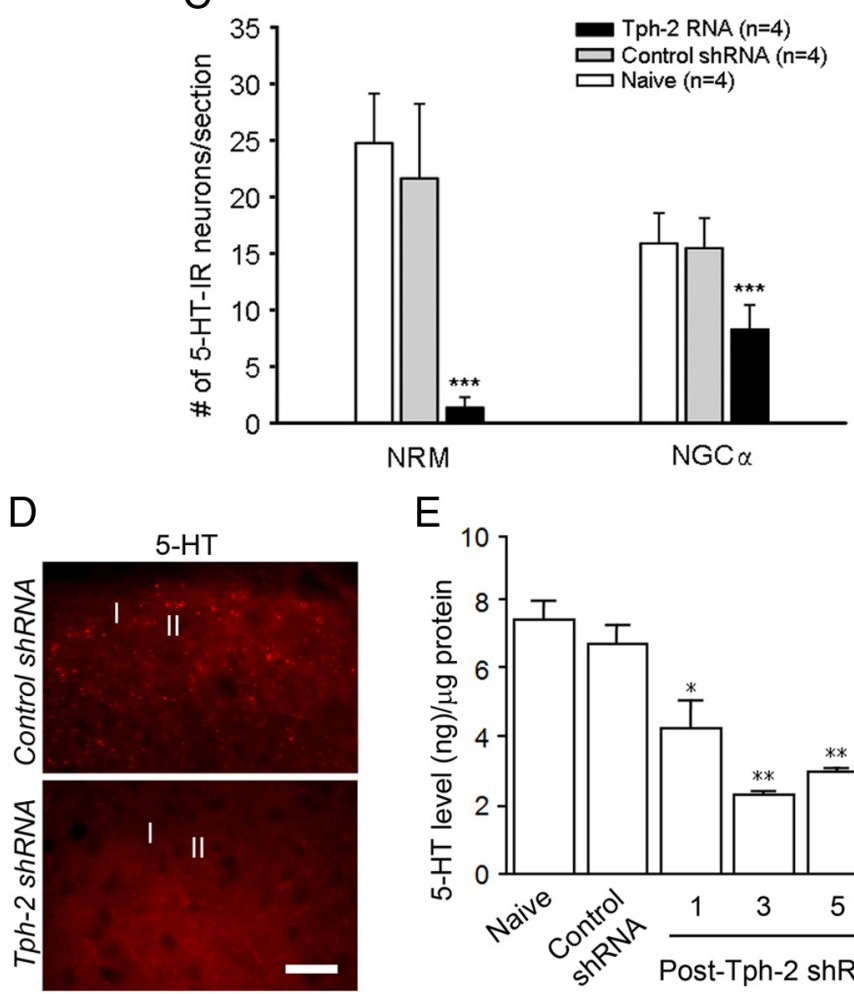

E

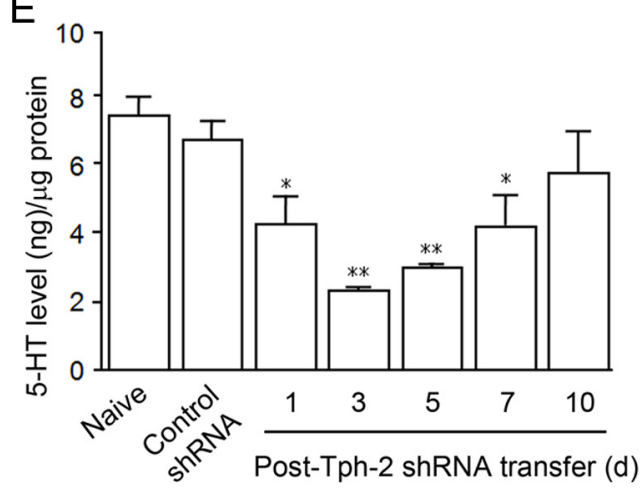

Figure 3. 5-HT expression and decrease in GFP-expressing RVM cells and the spinal dorsal horn. $A$, GFP expression in the NRM a medial area of the RVM, at $3 \mathrm{~d}$ after control gene transfer $(\boldsymbol{A a})$. High magnification of images from the inset in $\boldsymbol{A a}$ shows GFP expression $(\boldsymbol{A} \boldsymbol{b})$ and 5-HT immunoreactivity $(\boldsymbol{A c})$ in NRM neurons (arrowheads). Normal 5-HT immunoreactivity is present in GFP-expressing neurons (arrowheads, $\boldsymbol{A d}$ ). $\boldsymbol{B}$, GFP-expressing neurons in the NRM at $3 \mathrm{~d}$ after transfer of plasmid of Tph-2 shRNA $(\mathbf{B a})$. High magnification of images from the inset in $\boldsymbol{B} \boldsymbol{a}$ shows GFP expression in NRM neurons (Bb) and sparse 5-HTimmunoreactive profiles (arrows) but not cell bodies in the NRM area $(\boldsymbol{B C})$. Double image indicates the loss of 5 -HT immunostaining in GFP-expressing neurons in the RVM at $3 \mathrm{~d}$ after transfer of plasmid of Tph-2 shRNA (Bd). C, Summary data of the effect of shRNA transfer at $3 \mathrm{~d}$ on $5-\mathrm{HT}$ immunoreactivity in the NRM and $\mathrm{NGC} \alpha$, a medial and a lateral area, respectively. Tph- 2 shRNA significantly reduces 5-HT immunoreactivity in the RVM compared to control shRNA, especially in the NRM than the NGC $\alpha$ ( $n=4$ per group ${ }^{* * *} p<0.001$, vs control shRNA). D, Normal distribution and reduction of 5-HT-labeled fibers in superficial spinal cord (lamina I and II) at $3 \mathrm{~d}$ after control (top) or Tph-2 shRNA (bottom panel) treatment. $\boldsymbol{E}$, ELISA showing time course-dependent decrease of tissue 5-HT concentration in spinal dorsal horn after gene transfer $\left[n=3,{ }^{*} p<0.05 ;{ }^{* *} p<0.01\right.$, vs control shRNA $\left.(n=3)\right]$. Scale bars: $200 \mu \mathrm{m}(\boldsymbol{A a}, \boldsymbol{B a}), 50 \mu \mathrm{m}(\boldsymbol{A} \boldsymbol{b}-\boldsymbol{A d}, \boldsymbol{B} \boldsymbol{b}-\boldsymbol{B d}), 20 \mu \mathrm{m}(\boldsymbol{D})$.

gene transfer and from $1 \mathrm{~d}$ to $7 \mathrm{~d}$ after gene transfer (Fig. $4 A, B$ ). Consistent with a study in which $l m \times 1 b$ knock-out mice lacking hindbrain 5-HT neurons exhibited normal locomotor activity (Zhao et al., 2006), analysis of rotarod data did not demonstrate any significant difference in latencies between the both groups from $1 \mathrm{~d}$ to $7 \mathrm{~d}$ after gene transfer (Fig. 4C), ruling out a possible effect of descending 5-HT deletion on spinal motorneurons involved in hindpaw movement. These results suggest that the RVM 5-HT system may not be involved in descending modulation in response to acute painful stimuli. 
A

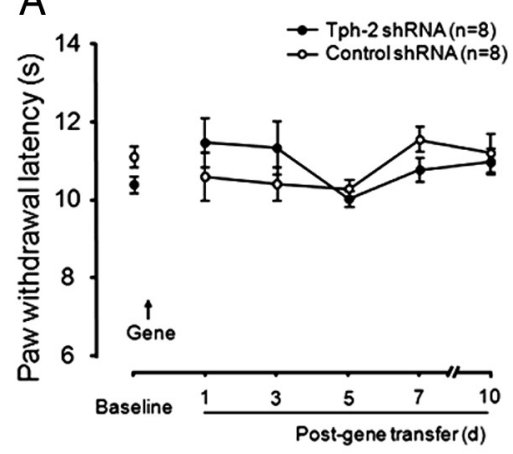

$\mathrm{C}$

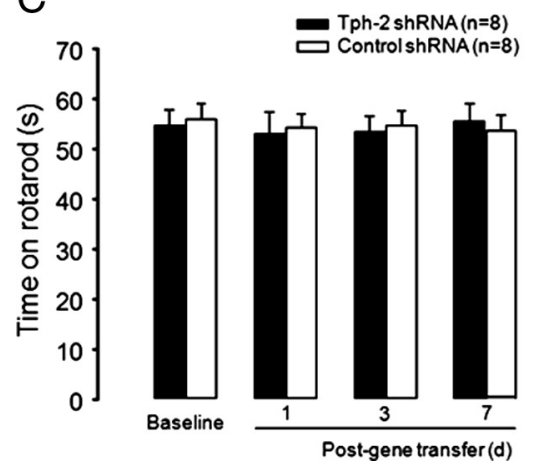

B
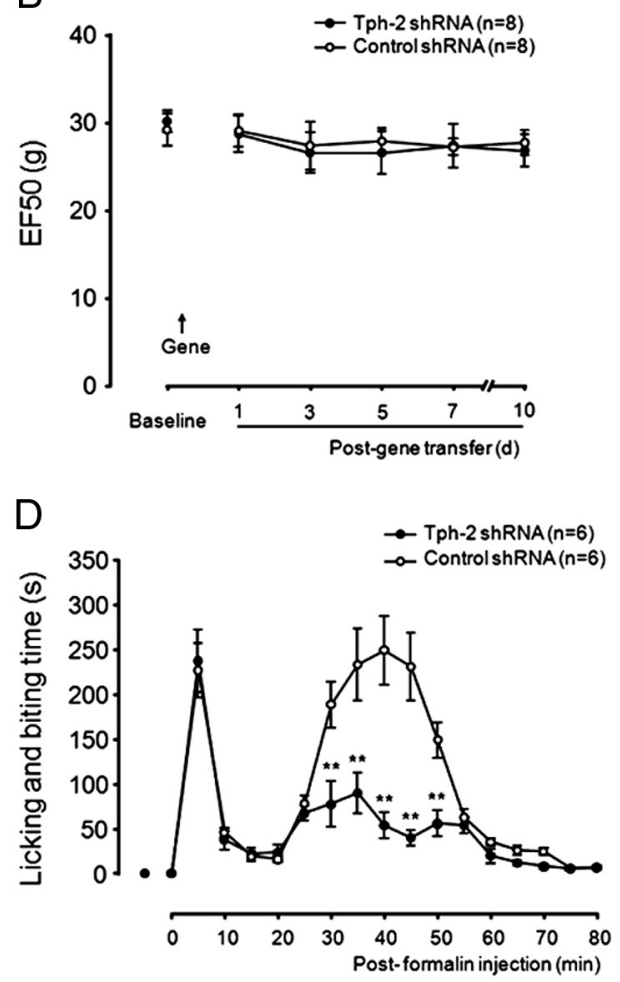

Figure 4. Behavioral responses to acute pain, hindpaw injection of formalin in rats with gene transfer. The RVM 5-HT system is not required for acute pain responses to thermal $(\boldsymbol{A})$ and mechanical $(\boldsymbol{B})$ stimuli. Animal mobility is measured using the rotarod test. No difference between both groups is observed, ruling out a potential motor impair in descending 5 -HT-deleted rats $(\boldsymbol{C})$. The descending depletion of Tph-2 significantly attenuates of the spontaneous nocifensive responses in the second phase but not the first phase after unilateral hindpaw injection of formalin $(\boldsymbol{D}) .{ }^{* *} p<0.01$, Tph-2 shRNA versus control shRNA-treated group.

of gene transfer from $14 \mathrm{~d}$ after SNL. There were no significant differences in baseline of thermal and mechanical sensitivity among all groups before gene transfer (Fig. 5A-D). After unilateral intraplantar CFA injection at $3 \mathrm{~d}$ following gene transfer, there was a similar level of hindpaw edema in rats treated either with the control $(n=8)$ or Tph- 2 shRNA ( $n=$ 8) from $1 \mathrm{~d}$ to $7 \mathrm{~d}$ after gene transfer (data not shown), indicating an equal development of peripheral inflammation. However, control shRNA-treated animals exhibited significant decreases in the thermal withdrawal latency (Fig. 5A) and mechanical withdrawal threshold $\left(\mathrm{EF}_{50}\right)$ (Fig. $5 B$ ) from $6 \mathrm{~h}$ to $5 \mathrm{~d}$ measured after CFA administration compared with the same pretreatment with control shRNA $(n=5)$ rats and without CFA injection. In contrast, the 5-HT depletion protected animals from the later development of thermal hyperalgesia (Fig. 5A) and mechanical hyperalgesia and allodynia (Fig. $5 B)$ at 1 and $3 \mathrm{~d}$ after CFA injection $(p<$ 0.05 vs group with treatment of control gene plus CFA), which is equivalent to 4-6 d following gene transfer, but did not block the development of hyperalgesia or partially reduced mechanical hyperalgesia and allodynia at an earlier time point $(6 \mathrm{~h})$ and a later $5 \mathrm{~d}$ time point $(p>0.05)$, compared with control gene treatment

Reduction of formalin-induced second phase of the nocifensive response in the intra-RVM 5-HT-depleted rats To determine the effects of the intra-RVM 5-HT depletion on the development of persistent pain, we performed the formalin test in both control and 5-HT-depleted rats at $3 \mathrm{~d}$ after gene transfer, at a time when RVM 5-HT depletion reached a peak. Spontaneous nocifensive responses (licking and scratching) are monitored in two phases before and after injection of a diluted formalin into the intraplantar region of the hindpaw. Consistent with acute pain being intact in the 5-HT-depleted rats, we found that the first phase (0-10 min) of pain response was not affected in control $(n=6)$ and the 5-HT-deleted $(n=6)$ animals. However, pain behavior in the second phase was significant attenuated in the 5-HT-deleted group (all $p<0.01$ at 30-50 min after formalin injection), compared with that in control-treated rats (Fig. 4D), suggesting that the RVM-spinal 5-HT system is implicated in descending pain facilitation involving central mechanisms (Wei et al., 2001). These results also further support the previous finding by Green et al. (2000), which has shown that a facilitatory role of spinal 5-HT3 receptor in formalin-induced nocifensive responses.

\section{Attenuation of thermal hyperalgesia and mechanical allodynia after inflammation and nerve injury in the intra-RVM 5-HT-depleted rats}

To determine the effects of RVM 5-HT depletion on behavioral responses to noxious stimuli in rats with persistent pain, we examined responses in a model of inflammatory pain induced by intraplantar injection of CFA from $3 \mathrm{~d}$ after gene transfer pretreatment and in a model of neuropathic pain by posttreatment and CFA injection (Fig. $5 A, B$ ). At 1 d, CFA-induced hyperalgesia is partially attenuated when compared to baseline shown in Tph-2 shRNA-treated rats without CFA injection $(p<0.05, n=5)$. At $1 \mathrm{~d}$, the development of mechanical hyperalgesia and allodynia was also totally blocked after CFA injection in the 5-HT-depleted animals (Fig. 5B). These results suggest that the descending 5-HT system originating from the RVM may play a progressive role in maintenance but not initiation of the development of persistent pain after peripheral inflammation. In another experimental pain model, nerve injury by SNL produced long-lasting and stable thermal hyperalgesia (Fig. 5C) and mechanical hyperalgesia and allodynia (Fig. 5D) for $14 \mathrm{~d}$ before and after posttreatment with control shRNA $(n=6)$. However, the posttreatment with Tph-2 shRNA significantly reduced thermal hyperalgesia from $1 \mathrm{~d}$ to $7 \mathrm{~d}$ after gene transfer $(n=8, p<0.05$ at $1 \mathrm{~d}$ and $7 \mathrm{~d}, p<0.001$ at 3 and $5 \mathrm{~d}$, vs control shRNA) (Fig. $5 C$ ). A similar time course of attenuation of mechanical hyperalgesia and allodynia was also observed (Fig. 5D). Furthermore, in the control shRNA-treated group, Western blot also showed that the tissue Tph-2 level was significantly increased in the RVM at $14 \mathrm{~d}$ after nerve injury (by $211.0 \pm 35.8 \%$ of the naive group without SNL and gene transfer, $p<0.05$ vs sham, $n=3$ per group), compared with that in sham rats without SNL (by $115.0 \pm 17.3 \%$ of naive, $p>0.05$ vs naive, $n=3$ ) (Fig. $5 E$ ). However, at $3 \mathrm{~d}$ after posttreatment with Tph-2 shRNA, SNL-induced Tph-2 elevation was totally blocked $14 \mathrm{~d}$ after nerve injury $(82.3 \pm 16.7 \%$ of naive, $p<0.05$ vs control shRNA, $n=3 ; p>0.05$ vs naive) (Fig. $5 E$ ), suggesting that the depletion of 5-HT by RNAi in the RVM is responsible for attenuation of nerve injury-induced pain hypersensitivity. Our results also suggest that $\sim 40 \%$ reduction of dorsal horn 5 -HT level at $1 \mathrm{~d}$ 
and $7 \mathrm{~d}$ following Tph-2 deletion in the RVM is enough to significantly prevent animals from maintaining hyperalgesia and allodynia after nerve injury. Thus, the RVM 5-HT system is likely involved in descending facilitation underlying the maintenance of inflammatory or neuropathic pain states. This is consistent with previous findings that the blocking of neuronal activity by intra-RVM injection of lidocaine or the lesioning of MOR-containing neurons in the RVM selectively attenuates maintenance of neuropathic pain states (Porreca et al., 2001; Burgess et al., 2002).

\section{Blockade of intra-RVM injection of BDNF-induced descending pain facilitation by the intra-RVM 5-HT depletion}

In our previous study (Guo et al., 2006), intra-RVM injection of BDNF (100 fmol) in naive rats produced a reversible and mild thermal hyperalgesia for $1 \mathrm{~d}$ and was dependent on NMDA receptor mechanisms, suggesting that BDNF can evoke descending pain facilitation. In view of evidence that TrkB, the receptor for BDNF, is widely expressed in RVM neurons (Guo et al., 2006), and that TrkB mRNA and 5-HT coexist in RVM-spinal neurons (King et al., 1999), it is reasonable to speculate that BDNF-induced descending facilitation may be mediated by spinal 5-HT released from activated RVM neurons expressing TrkB. To confirm this hypothesis, we first injected FG, a retrograde dye, into the superficial dorsal horn at lumber enlargement, and immunostaining showed FG-labeled spinally projection neurons in the RVM (Fig. 6A) at 1 week after FG injection. Further combination with double immunostaining for TrkB and 5-HT showed that the majority of FG-labeled RVM neurons expressed TrkB and were also immunoreactive to 5-HT (Fig. 6A), suggesting that the RVM-spinal 5-HT system may mediate BDNF-induced descending pain facilitation. Thus, to examine the possible role of the descending 5-HT pathway in descending pain facilitation, mimicking the role of the descending 5-HT system in persistent pain after tissue and nerve injury, genetransferred rats received a single microinjection of BDNF ( $100 \mathrm{fmol}$ ) or vehicle into the RVM. Paw withdrawal latency was monitored to evaluate the effects of RVM 5-HT depletion on BDNF-induced descending pain facilitation. Consistent with our previous observation (Guo et al., 2006), exogenous BDNF (100 fmol) in the RVM produced mild thermal hyperalgesia up to $1 \mathrm{~d}$ (Fig. $6 B$ ) compared with vehicle administration in control gene-treated animals ( $p<0.05$ at $2-24 \mathrm{~h}, n=6-8$ per group). The same dose of
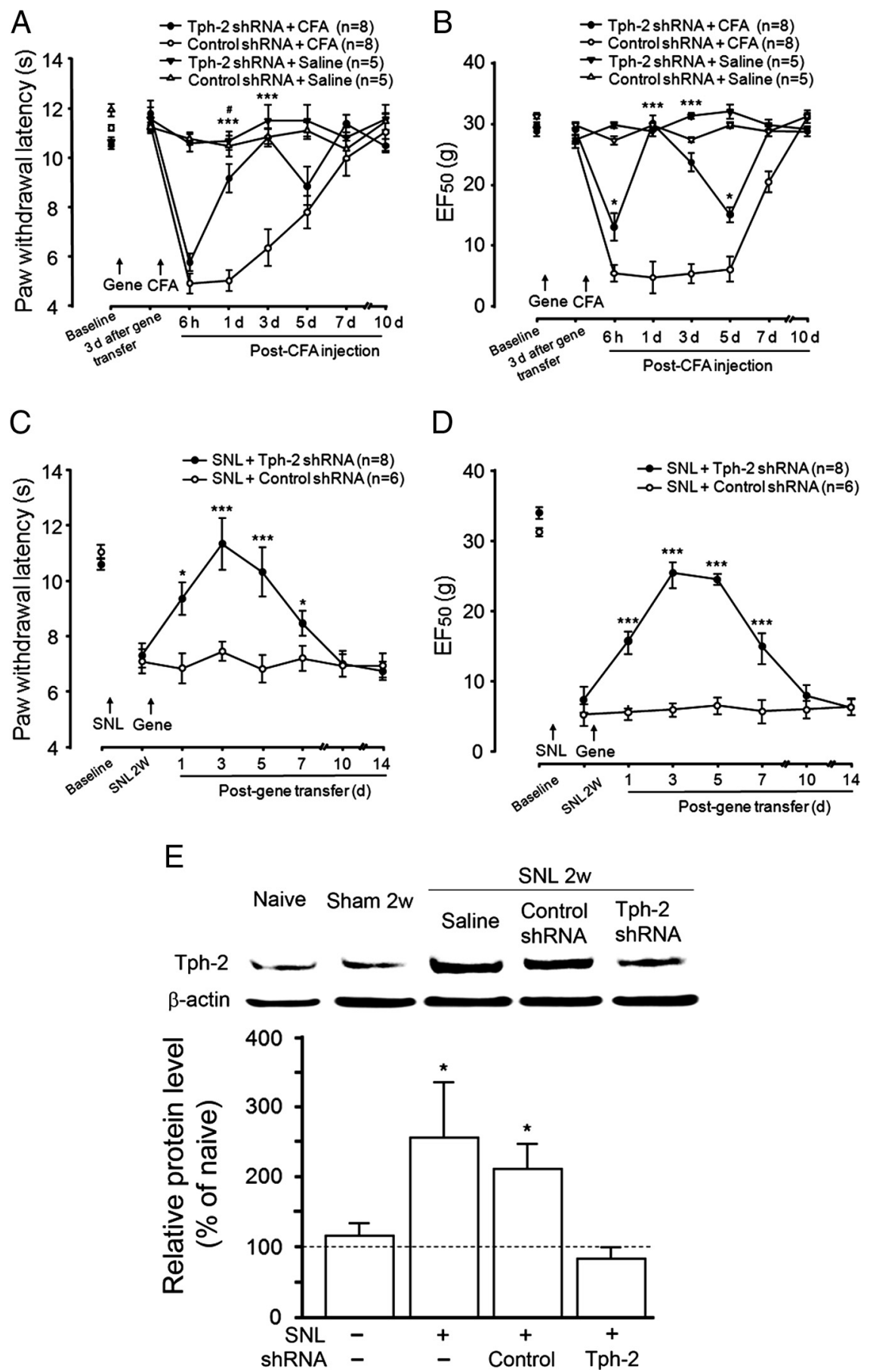

Figure 5. Attenuation of behavioral hypersensitivity and enhanced Tph-2 in the RVM in rats with RVM 5-HT depletion after hindpaw inflammation and nerve injury. Significant attenuations of thermal hyperalgesia at $1-3 \mathrm{~d}$ but not $6 \mathrm{~h}$ and $5 \mathrm{~d}$ after CFA-induced peripheral inflammation by pretreatment of the 5 -HT depletion compared to the control shRNA-treated rats $(\boldsymbol{A})$. RVM 5-HT depletion completely eliminates or attenuates of mechanical hyperalgesia and allodynia at $1-3 \mathrm{~d}$ or $6 \mathrm{~h}$ and $5 \mathrm{~d}$, respectively, in rats with inflammation compared to control treatment $(\boldsymbol{B})$. Long-lasting reduction of thermal hyperalgesia $(\boldsymbol{C})$ and mechanical hyperalgesia and allodynia (D) from $1 \mathrm{~d}$ to $7 \mathrm{~d}$ by posttreatment of the 5 -HT deletion followed $14 \mathrm{~d}$ nerve injury. $\boldsymbol{E}$, Western blots showing that Tph-2 protein levels in the RVM tissue are significantly upregulated at 2 weeks after nerve injury compared with that in naive animals. Posttreatment with Tph-2 shRNA completely eliminates SNL-induced increase of Tph-2 protein levels in the RVM at $3 \mathrm{~d}$ after gene transfer compared to control treatment. For $\boldsymbol{A}-\boldsymbol{D},{ }^{*} p<0.05 ;{ }^{* * *} p<0.001$, versus control shRNA; ${ }^{*} p<0.05$ versus hindpaw saline in Tph-2 shRNA-treated group. For $\boldsymbol{E}$, all ${ }^{*} p<0.05$ versus sham.

BDNF also induced significantly mechanical allodynia at $4-6 \mathrm{~h}$ after intra-RVM microinjection compared to ACSF in control shRNAtreated groups ( $p<0.05, n=4-8$ per group) (Fig. $6 C$ ). However, the RVM 5-HT depletion not only completely blocked BDNF- 
A

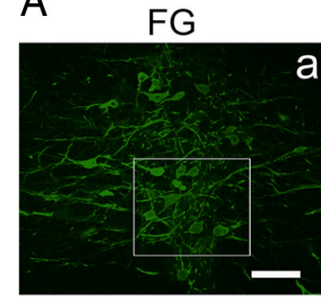

TrkB

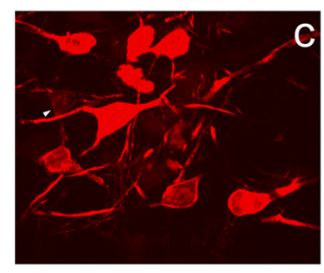

$5-\mathrm{HT}$

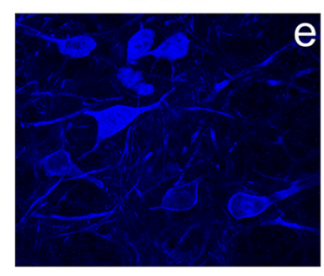

FG

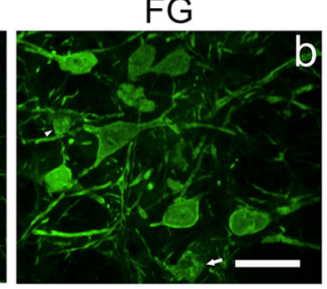

TrkB/FG

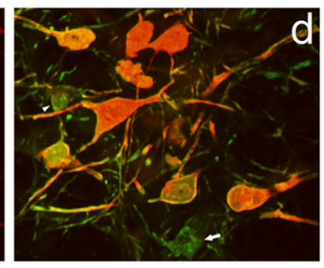

TrkB/5-HT/FG

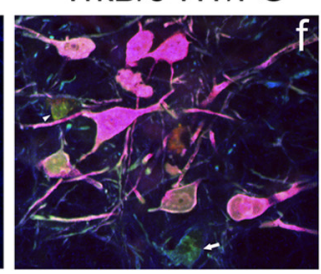

B

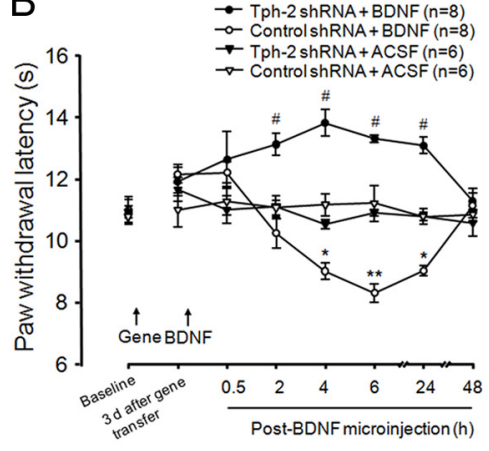

C
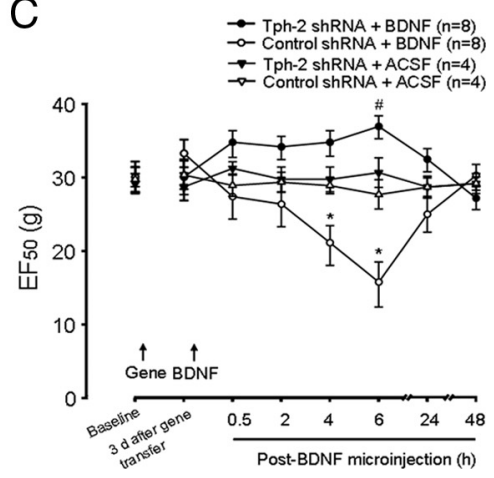

Figure 6. The colocalization of TrkB and 5-HT in RVM neurons projecting to spinal dorsal horn and the elimination of intra-RVM BDNF-induced hyperalgesia by the descending $5-H T$ depletion. $\boldsymbol{A a}$, Distribution of retrograde tracer $\mathrm{FG}$ expression in neurons of the RVM area at $7 \mathrm{~d}$ after microinjection in the lumbar spinal dorsal horn. $\boldsymbol{A} \boldsymbol{b}$, A high-magnification view of the inset outlined in $\boldsymbol{A} \boldsymbol{a}$ shows the spinal projecting RVM neurons and their dendrites expressing $\mathrm{FG}$. $\boldsymbol{A c}-\boldsymbol{A f}$, Combination of double immunostaining with retrograde FG tracking further demonstrates the expression of TrkB $(\boldsymbol{A c})$ in the most FG-labeled RVM neurons ( $\boldsymbol{A d})$ and the colocalization of 5-HT immunoreactivity (IR, $\boldsymbol{A e}$ ) with TrkB-IR in the FG-labeled neurons in the RVM (Af). Scale bars: $200 \mu \mathrm{m}(\boldsymbol{A a})$, $50 \mu \mathrm{m}(\boldsymbol{A} \boldsymbol{b}-\boldsymbol{A} \boldsymbol{f})$. B, Microinjection of BDNF (100 fmol) induces a prolonged thermal hyperalgesia in control gene-treated but not Tph-2 shRNA-treated rats. $C$, The same dose of BDNF results in mechanical allodynia in control but not Tph-2 shRNA-treated animals at $4-6 \mathrm{~h}$ after gene transfer in the RVM. ${ }^{*} p<0.05 ;{ }^{* *} p<0.01$, BDNF versus ACSF in control shRNA-treated rats; ${ }^{*} p<$ 0.05, BDNF versus ACSF in Tph-2 shRNA-treated animals.

induced thermal hyperalgesia and mechanical allodynia, it switched the effect of BDNF on descending modulation from facilitation to inhibition when compared with control treatment $(p<0.05, n=8$ per group) (Fig. 6B,C), which is only observed after a higher pharmacological dose administration of BDNF in the RVM in intact animals (Guo et al., 2006). These findings indicate that the descending 5-HT system is pronociceptive after activation of the RVM BDNF-TrkB signaling pathway in normal animals and mediates endogenous BDNF-induced descending pain facilitation, which is known to be involved in molecular mechanisms underlying the development of persistent pain after inflammation (Guo et al., 2006). Thus, the depletion of descending 5-HT from the RVM abolished BDNF-induced hyperalgesia and allodynia in the present study, further supporting our hypothesis that spinal 5-HT systems mediate BDNF-induced descending pain facilitation.

\section{Descending pain inhibition induced by intra-RVM electrical} stimulation is eliminated by the intra-RVM 5-HT depletion Earlier studies (Yaksh and Wilson, 1979; Hammond and Yaksh, 1984; Hammond et al., 1985) indicated that global excitation of RVM neurons by strong ES $(50-150 \mu \mathrm{A})$ induced temporary descending inhibition in behavioral nociceptive tests and increased spinal 5-HT release. These behavioral effects were attenuated by pretreatment of the intrathecal injection of the 5-HT1 and 5-HT2 receptor antagonist methysergide, suggesting the in- volvement of activated RVM neurons, including 5-HT-containing neurons in the ES-induced descending inhibition. We questioned whether 5-HT in descending projecting RVM neurons was sufficient to mediate the ES-evoked descending inhibition. To test this possibility, we used the same protocol described (Hammond and Yaksh, 1984) in the shRNA-treated animals to examine effects of RVM 5-HT depletion on ES-induced descending inhibition. As shown in Figure 7, ES in the RVM induced a significant increase in paw withdrawal latency, a measure of descending pain inhibition, during ES and at least $5 \mathrm{~min}$ after $\mathrm{ES}$ in naive $(n=4)$ or control shRNA-treated rats $(n=6)$ in a similar manner (Fig. 7). However, 5-HT depletion in the RVM completely eliminated ES-induced descending inhibition $3 \mathrm{~d}$ after gene transfer $(p<0.05, n=6$ vs control group). These data suggest that global RVM neurons are activated by ES, some of their descending terminals release 5 -HT in the spinal cord, and this neurotransmitter contributes to ES-induced descending inhibition of spinal nociceptive processing.

Descending pain inhibition induced by intra-RVM opioid receptor activation is not changed by the intra-RVM 5-HT depletion

Interestingly, Hurley and colleagues (Hurley and Hammond, 2001; Hurley et al., 2003) reported that microinjection of the $\mu$-opioid receptor agonist DAMGO (3-40 ng) in the RVM produced dose-dependent increases in paw withdrawal latency in normal rats, which was prevented by intrathecal pretreatment of methysergide, suggesting that DAMGO-induced descending pain inhibition is also partially mediated by descending 5-HT and its receptors in the spinal cord. Therefore, we further investigated the effects of RVM 5-HT depletion on descending pain inhibition produced by DAMGO. At $3 \mathrm{~d}$ after gene transfer, compared to ACSF, microinjection of DAMGO (40 or $100 \mathrm{nmol}$ ) in the RVM produced a transient and dose-dependent inhibition in thermal (Fig. $8 A$ ) and mechanical (ANOVA, $p<0.05$ at $15 \mathrm{~min}, 40 \mathrm{vs} 100 \mathrm{nmol}$ ) (Fig. 8 B) sensitivity, which did not differ between the 5-HT-depleted $(n=6-8)$ and control $(n=4-8)$ groups $(p>0.05)$ (Fig. $8 A, B)$. Thus, in contrast to that in ES-evoked descending inhibition, release of 5-HT from the descending pathway in the RVM was insufficient to mediate intra-RVM MOR activation-induced analgesia. Consistent with behaviorally intact descending inhibition induced by intra-RVM DAMGO, immunostaining also demonstrated normal expression of MOR in GFP-expressing neurons in the RVM of Tph-2-depleted rats compared with that in the control group (data not shown). Collectively, these findings suggest that RVM MOR activation-induced analgesia is not mediated via descending 5-HT mechanisms; and also more likely, 5-HTmediated descending inhibition after neuronal activation in the RVM may be partially dependent on activation of other opioid receptor subtypes, such as the KOR. For example, in behavioral 


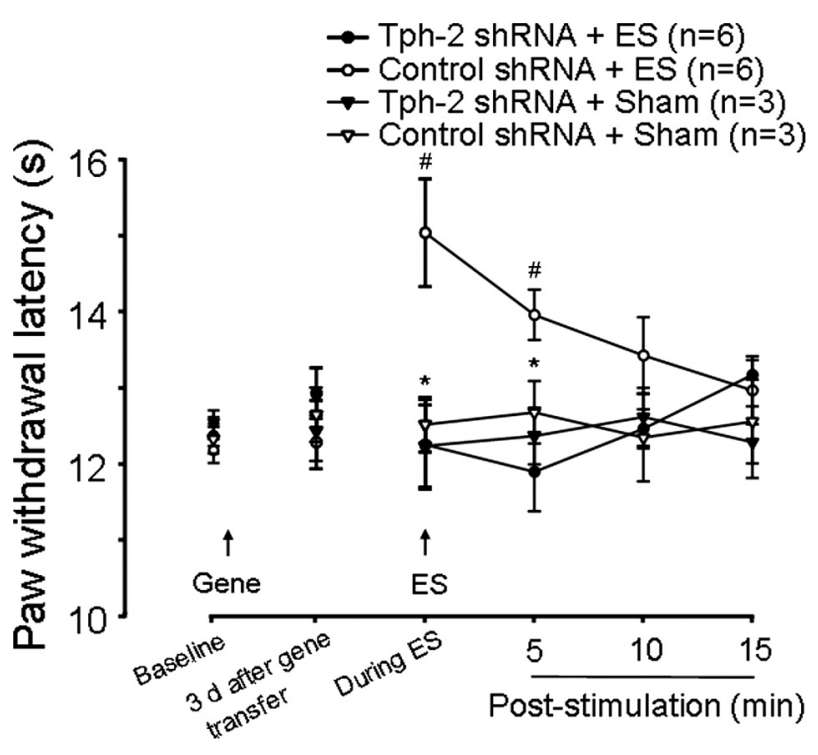

Figure 7. Descending 5-HT depletion blocks intra-RVM ES-induced descending pain inhibition. Intra-RVM ES significantly induces transient increases of paw withdrawal latency, a measure of analgesia, during ES and at $5 \mathrm{~min}$ after $E S$ compared to baseline at $3 \mathrm{~d}$ after gene transfer $\left({ }^{\#} p<0.05\right)$ in control shRNA-treated rats, which is completely eliminated in Tph-2 shRNAtreated rats. ${ }^{*} p<0.05$; Tph-2 shRNA versus control shRNA treatment. Sham treatment for ES does not change thermal sensitivities in both control and Tph-2 shRNA-treated rats.

experiments, high doses of intra-RVM KOR agonist U69593 induce analgesia (Ackley et al., 2001), but lower doses produce a slight hyperalgesia (Ackley et al., 2001) or reduce antinociception produced by injection of DAMGO in the periaqueductal gray (PAG) (Pan et al., 1997). Morphological data have shown that many 5-HT-immunolabeled neurons in the RVM express KOR immunoreactivity (Kalyuzhny and Wessendorf, 1999). Wholecell patch recording has further revealed that KOR agonists produced an outward current in a subpopulation of RVM neurons (Pan et al., 1997; Vaughan et al., 1999; Ackley et al., 2001), which purportedly contain 5-HT (Pan et al., 1993). These studies suggest that the influences of KOR activation in the RVM on nociception could be dependent on RVM 5-HT mechanisms. To confirm this possibility, the effects of U69593 on nociception were examined in 5-HT-depleted animals. Consistent with a previous study (Ackley et al., 2001), microinjection of $3 \mathrm{nmol}$ U69593 in the RVM induced a time-dependent antinociception in the control group compared with vehicle treatment (Fig. $8 C, D)$. The transient increases in hindpaw thermal and mechanical threshold observed in the control group were not attenuated by depletion of 5-HT in the Tph-2 shRNA-treated group at 15 min after microinjection of U69593 (3 nmol) in the RVM ( $p=$ $0.555, n=6$ for each group) (Fig. $8 C, D)$. A low dose $(0.5 \mathrm{nmol})$ of U69593, which was shown to attenuate intra-PAG DAMGOinduced analgesia in the tail-flick test (Pan et al., 1997), also produced a significant increase in paw withdrawal latency and mechanical threshold in both 5-HT-depleted and control animals at 15 min compared with the baseline before the microinjection. In contrast, an even lower dose ( $0.1 \mathrm{nmol})$ of U69593 did not significantly change thermal and mechanical sensitivity before and at 15 min after microinjection in the RVM from both 5-HT-depleted [thermal: $11.27 \pm 0.15 \mathrm{~s}$ and $11.53 \pm 0.44 \mathrm{~s}(n=3$, $p>0.05)$; mechanical: $30.67 \pm 2.23 \mathrm{~g}$ and $29.28 \pm 3.82 \mathrm{~g}(n=3, p>$ $0.05)$ ] and control-treated [thermal: $10.87 \pm 0.21 \mathrm{~s}$ and $11.32 \pm$ $0.58 \mathrm{~s}(n=3, p>0.05)$; mechanical: $29.02 \pm 3.54 \mathrm{~g}$ and $29.70 \pm$ $4.20 \mathrm{~g}(n=3, p>0.05)]$ rats (data not shown). Furthermore, an- other KOR agonist U50488 was used to confirm the effects of descending 5-HT depletion on intra-RVM KOR activation-induced antinociception. Consistent with U69593, U50488 (5 or $20 \mathrm{nmol}$ ) produced significantly dose-dependent decreases in thermal (ANOVA, $p<0.05$ at 15 and $30 \mathrm{~min}, 5 \mathrm{vs} 20 \mathrm{nmol}$ ) (Fig. $8 \mathrm{E}$ ) and mechanical (ANOVA, $p<0.05$ at $15 \mathrm{~min}$ ) (Fig. $8 F$ ) sensitivity, which also did not differ between the 5-HT-depleted $(n=4-6)$ and control groups $(n=4-6)(p>0.05)$ (Fig. $8 E, F)$. Together, the presence of U69593 or U50488-induced antinociception in 5-HT-depleted animal suggests that the RVM 5-HT system in naive animals is not necessary for $\kappa$-opioid receptor-involved descending inhibition.

\section{Discussion}

In the present study, we used in vivo electroporation to transfer plasmids encoding Tph-2 shRNA or scrambled shRNA as a control into RVM neurons, thereby disrupting the function of the neuronal 5-HT synthesis enzyme by an RNAi mechanism, and depleting endogenous 5-HT in RVM 5-HT-containing neurons and their projection terminals in the spinal dorsal horn. Two features of our approach provide unique advantages for identification of descending 5-HT function not achievable with conventional pharmacological neurotoxins and genomic deletion. First, selective disruption of descending 5-HT function is achieved by a reversible downregulation of the 5-HT molecule in RVM 5-HTcontaining neurons with treatment of Tph-2 RNAi. There is no loss of RVM neurons containing 5-HT as with the selective serotonergic neurotoxin 5,7-DHT (Wei et al., 1999a) or with genomic knock-out of the transcription factor $L m x 1 b$, which is essential for the development of 5-HT-containing neurons in the hindbrain (Zhao et al., 2007). It should be noted that the specific loss of central 5-HT-containing neurons not only removes 5-HT but also deletes other colocalized neurotransmitters in their functional terminals in the spinal cord and a variety of receptors expressed on their somatodendritic fields in the RVM, which contributes to descending pain facilitation or inhibition (Basbaum and Fields, 1984; Porreca et al., 2002; Dubner and Ren, 2004; Fields et al., 2006). Second, in LMX1b knock-out mice, the loss of 5-HT neurons is not limited to the RVM region but spreads to other brainstem serotonergic nuclei that are known to send ascending projections to forebrain structures that are involved in the modulation of the sensory and affective components of pain (Wei et al., 2001; Johansen and Fields, 2004). For example, some groups (Rahman et al., 2006; Géranton et al., 2008) have found that selective lesion of spinal 5-HT fibers by 5,7-DHT reduces central sensitization and attenuates mechanical allodynia after tissue and nerve injury but not thermal hyperalgesia after inflammation. In contrast, the same lesions of spinal 5-HT fibers (Millan et al., 1997; Pertovaara et al., 2001) or supraspinal 5-HTcontaining neurons in the RVM (Wei et al., 1999a) and hindbrain (Zhao et al., 2007) enhance thermal hyperalgesia after inflammation. Given the controversial results in these studies and integrated impairment of descending 5-HT-containing neurons or fibers, the role of descending 5-HT but not 5-HT-containing neurons in the development of thermal and mechanical hypersensitivity after inflammation and nerve injury requires reevaluation. In the present study, the selective and effective depletion of 5-HT was successfully achieved without neuronal lesion and limited to the RVM and the spinal cord where raphe-spinal neurons terminate. We observed the time-dependent attenuation of both thermal hyperalgesia and mechanical allodynia after tissue or nerve injury, which matches the duration of RVM Tph-2 depletion and spinal 5-HT reduction. Using this molecular depletion 

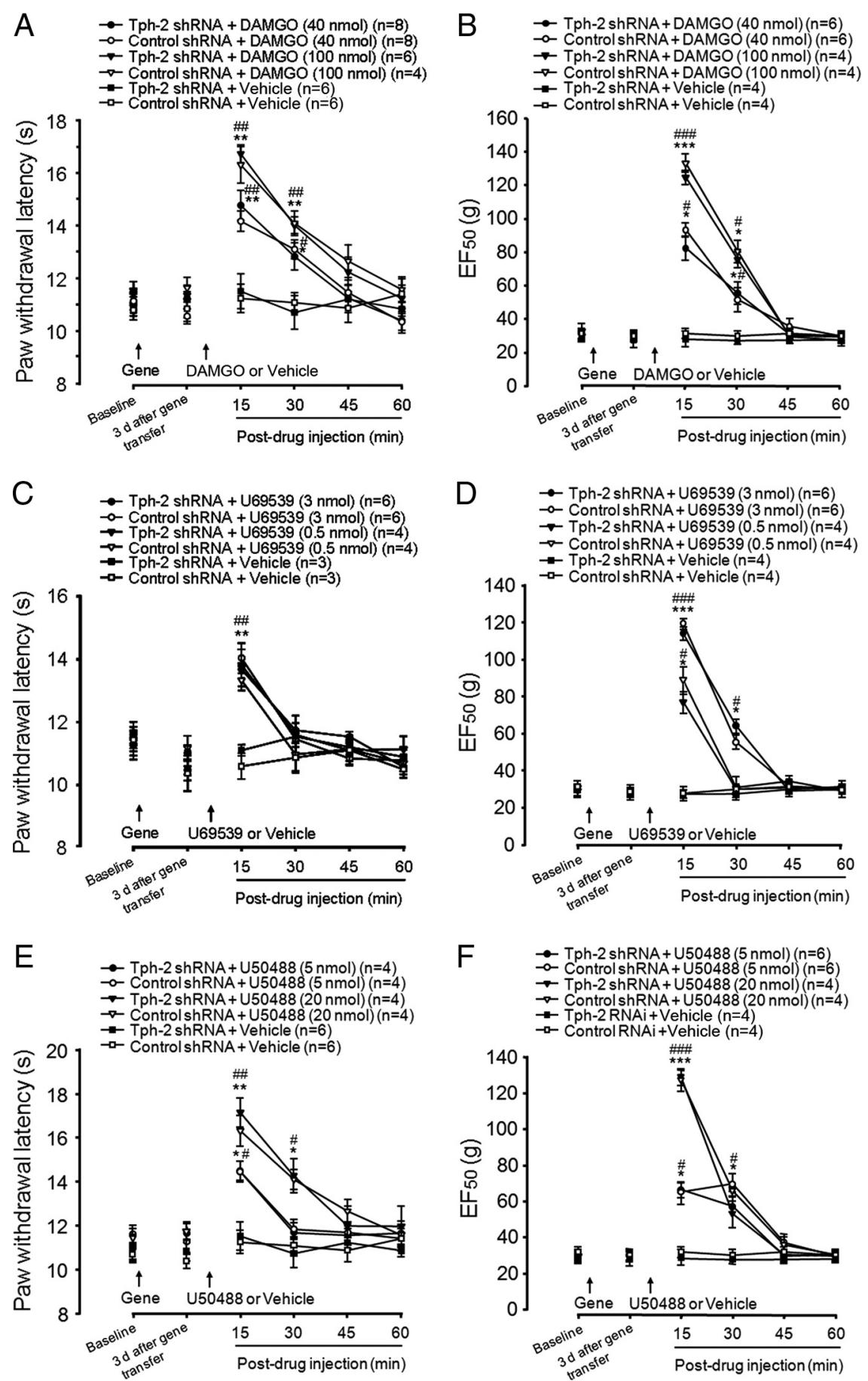

Figure 8. Descending 5-HT depletion does not change opioid-induced descending inhibition in acute pain. $\boldsymbol{A}, \boldsymbol{B}$, IntraRVM injection of MOR agonist DAMGO produces dose-dependent increases of thermal $(\boldsymbol{A})$ and mechanical $(\boldsymbol{B})$ thresholds in hindpaw of both control shRNA- and Tph-2 shRNA-treated rats compared to vehicle. There is no difference in intra-RVM DAMG0-induced analgesia between Tph-2 shRNA- and control shRNA-treated groups at $3 \mathrm{~d}$ after gene transfer. ${ }^{*} p<0.05$; ${ }^{* *} p<0.01$, DAMG0 versus vehicle in Tph-2 shRNA-treated rats; ${ }^{\#} p<0.05,{ }^{\# \#} p<0.01$; DAMG0 versus vehicle in control shRNA-treated rats. $\boldsymbol{C}, \boldsymbol{D}$, The analgesic effects produced by microinjection of the KOR agonist $U 69593$ ( 5 and $20 \mathrm{nmol}$ ) in the RVM on thermal $(\boldsymbol{C})$ and mechanical $(\boldsymbol{D})$ sensitivity were measured between Tph-2 shRNA-treated and control shRNAtreated groups. ${ }^{*} p<0.05,{ }^{* *} p<0.01,{ }^{* * *} p<0.001$; U69593 versus vehicle in Tph-2 shRNA-treated rats; ${ }^{*} p<0.05$, $\# p<0.01 ;$ U69593 versus vehicle in control shRNA-treated rats. $\boldsymbol{E}, \boldsymbol{F}$, Another KOR agonist U50488 after microinjection in the RVM induced similar dose-dependent increases of hindpaw thermal $(\boldsymbol{E})$ and mechanical $(\boldsymbol{F})$ thresholds in both control shRNA and Tph-2 shRNA-treated rats. ${ }^{*} p<0.05,{ }^{* *} p<0.01$; U50488 versus vehicle in Tph-2 shRNA-treated rats; ${ }^{\#} p<$ $0.05,{ }^{\# \#} p<0.01,{ }^{\# \#} p<0.001 ;$ U 50488 versus vehicle in control shRNA-treated rats. technique, we show for the first time that descending 5-HT from the RVM plays a critical role in enhanced descending pain facilitation during persistent pain states, supporting the conclusion that RVM 5 -HT is at least partially required for development and maintenance of persistent pain states.

The behavioral nociceptive responses mediated by descending 5-HT are dependent on the activation of diverse 5-HT subtype receptors. All 5-HT receptor subtypes (5-HT1-7) are expressed in the spinal dorsal horn (Hamon and Bourgoin, 1999; Jeong et al., 2004) and exert a modulatory effect on spinal nociceptive responses. The antinociceptive influence of 5-HT appears to be mediated primarily by spinal 5 -HT1 and 5-HT2 receptors (Hamon and Bourgoin, 1999; Millan, 2002). Although the activation of the 5-HT3 receptor has been reported to inhibit nociception (Glaum et al., 1990; Alhaider et al., 1991; Paul et al., 2001), it also has been noted to play a facilitatory role in formalin-induced nocifensive responses (Green et al., 2000; Zeitz et al., 2002). Accumulating evidence indicates that the blockade of the 5-HT3 receptor specifically attenuates pronociceptive actions of 5-HT in persistent pain models (Oatway et al., 2004) and may include a spinal-bulbo-spinal loop (Suzuki et al., 2002; Zeitz et al., 2002; Svensson et al., 2006). Recently, we have shown that intrathecally administered 5-HT3 receptor agonists produce pain hypersensitivity via a signaling cascade that includes the activation of glia, the release of the cytokine IL- $1 \beta$ and activation of its receptor on neurons (our unpublished observations). Consistent with the above data, 5-HT3A knock-out mice showed a dramatic decrease in the second phase of the formalininduced nocifensive response (Kayser et al., 2007). In the present study, depletion of the descending 5-HT system did not affect basal expression of thermal and mechanical nociception, but significantly attenuated formalin-induced spontaneous nocifensive responses, inflammation-induced hyperalgesia and allodynia, and the maintenance of neuropathic pain states. Regarding the reduction of spinal 5-HT3 receptor activation after intra-RVM 5-HT depletion, our data further support previous work and the hypothesis that descending 5-HT and spinal 5-HT3 receptor activation contribute to enhanced descending pain facilitation after tissue and nerve injury. Interestingly, recent studies have shown upregulation of 5-HT3 receptor expression in DRG neurons and tri- 
geminal nuclei after inflammation (Liu et al., 2005) and nerve ligation (Doucet et al., 2007). The present study is the first demonstration that Tph-2 protein in the RVM tissue is upregulated after nerve injury and its blockade attenuates nerve injuryinduced behavioral hypersensitivity. We suggest that the balance between RVM descending 5-HT inhibitory and facilitatory influences shifts toward pronociception after tissue and nerve injury via enhanced activation of pronociceptive 5-HT receptor subtypes, including the 5-HT3 receptor. It will be interesting to determine whether spinal 5-HT3 receptors underlie plasticity and/or sensitivity to residual 5-HT released after intra-RVM 5-HT depletion. The role of different spinal 5-HT receptor subtypes, including 5-HT3 receptor, in the development of spinal neuronal hyperexcitability clearly needs further investigation.

The majority of studies concerning the roles of neurochemically identified RVM neurons in spinal nociceptive modulation have focused on the electrophysiological properties of these neurons and its relationship with nocifensive responses to noxious stimulation. The descending facilitatory or inhibitory components appear to be mediated through recruitment of RVM ON or OFF cells, respectively (Fields and Heinricher, 1989). The activation of pronociceptive ON cells in the RVM has been reported to correlate with behavioral hypersensitivity after noxious input (Fields and Heinricher, 1989) or nerve injury (Carlson et al., 2007). There is evidence suggesting that functional MOR expression predominates in ON cells in the RVM (Fields and Heinricher, 1989; Pan et al., 1990; Heinricher et al., 1994). Selective chemical deletion of MOR-containing cells in the RVM was found to attenuate maintenance of neuropathic pain (Porreca et al., 2001). Nearly half of the spinalprojecting 5-HT-containing neurons have been shown to express MOR (Wang and Wessendorf, 1999), and patchclamp recordings from rat RVM slice preparations also identified a proportion of spinally projecting 5-HT-containing neurons responsive to MOR agonists (Marinelli et al., 2002; Finnegan et al., 2004). It appears that at least some descending MOR-expressing ON cells use 5-HT as a neurotransmitter. The selective deletion of MOR-containing ON cells in the RVM would then reduce descending serotonergic facilitatory influences.

Based on the findings that RVM microinjection of morphine produced spinal 5-HT receptor-dependent analgesia (Hammond and Yaksh, 1984; Vasko et al., 1984; Dogrul et al., 2009), we further investigated whether descending 5-HT systems contribute to MOR activation-dependent descending inhibition using the highly selective MOR agonist, DAMGO. Surprisingly, 5-HT depletion in the RVM had no effect on descending pain inhibition induced by intra-RVM injection of DAMGO, consistent with recent electrophysiological data indicating a lack of activation of RVM 5-HT-containing neurons in morphine analgesia (Johannessen et al., 1982; Gao et al., 1998; Gao and Mason, 2000) and only a few (5\%) descending 5-HT-containing neurons responding to DAMGO in the RVM in acute pain (Zhang and Hammond, 2010). DAMGO microinjection has been reported to depress $\mathrm{ON}$ cell firing, indirectly exerting a net inhibitory effect on nociception (Heinricher et al., 1994). In addition, it has been known that 5-HT is exclusively present in some NEUTRAL cells and KOR is only expressed in OFF and NEUTRAL cells (Potrebic et al., 1994; Winkler et al., 2006). The majority of functionally KOR-expressing or KOR-labeled neurons are also immunoreactive for 5-HT (Pan et al., 1993; Kalyuzhny and Wessendorf, 1999; Ackley et al., 2001; Winkler et al., 2006). Activation of KOR in the RVM produces antinociception (Ackley et al., 2001) but attenu- ates intra-PAG DAMGO-induced anesthesia by inhibition of 5-HT neurons (Pan et al., 1997). However, our results indicate that intra-RVM KOR activation-induced antinociception is not impaired in the RVM 5-HT-depleted animals, suggesting that the RVM 5-HT system is not necessary for KOR-involved descending inhibition. Thus, depletion of 5-HT in RVM neurons would have the same inhibitory effect on ON cells as DAMGO or on NEUTRAL cells as U69593 or U50488, providing an explanation for the lack of any effect of 5-HT depletion on MOR or KOR effects. Thus, our findings indicate that descending 5-HT from the RVM is neither necessary nor sufficient for RVM MOR or KOR-mediated descending pain modulation in acute pain. Whether the RVM 5-HT system is involved in nonopioid descending pain inhibition or enhanced opioid descending anti-hyperalgesia after inflammation and nerve injury still requires further investigation.

In summary, the traditional concept of RVM 5-HT function in descending modulation has been mainly based on previous studies using the chemical damage of 5-HT-containing neurons in the RVM or 5-HT-containing fibers in the spinal cord and the pharmacological blockade of 5-HT receptor subtypes in the spinal dorsal horn. These studies suggest that the RVM 5-HT system is involved in a tonic descending inhibition of acute pain (Basbaum and Fields, 1984; Millan, 2002), or enhanced descending pain inhibition after inflammation (Wei et al., 1999; Zhao et al., 2007), or that subtypes of 5-HT receptors contribute to pronociception or antinociception (Millan, 2002; Suzuki et al., 2004; Lopez-Garcia, 2006). These controversial results do not demonstrate the integrated effects 5 -HT function in spinal pain processing and modulation because they do not reflect the unique effects of the descending neurotransmitter 5-HT itself. Utilization of combined regional gene targeting with RNA interference provides a timely tool for reevaluating the direct or integrated role of RVM 5-HT. In the present study, we selectively depleted 5-HT from RVM neurons and descending 5-HTcontaining axon terminals in the spinal dorsal horn. We found that the RVM 5-HT system was not involved in tonic descending inhibition and intra-RVM opioid-induced analgesia in acute pain. Importantly, our study identified that the RVM 5-HT system participated in enhanced descending pain facilitation, which was necessary for maintenance of hyperalgesia and allodynia after inflammation and nerve injury. We hypothesize that unique functional and structural plasticity of some spinal 5-HT receptor subtypes may be responsible for a switch in the balance of RVM 5-HT function resulting in enhanced descending facilitation during the development of persistent pain.

\section{References}

Ackley MA, Hurley RW, Virnich DE, Hammond DL (2001) A cellular mechanism for the antinociceptive effect of a kappa opioid receptor agonist. Pain 91:377-388.

Alhaider AA, Lei SZ, Wilcox GL (1991) Spinal 5-HT3 receptor-mediated antinociception: possible release of GABA. J Neurosci 11:1881-1888.

Basbaum AI, Fields HL (1984) Endogenous pain control systems: brainstem spinal pathways and endorphin circuitry. Annu Rev Neurosci 7:309-338.

Buhler AV, Choi J, Proudfit HK, Gebhart GF (2005) Neurotensin activation of the NTR1 on spinally-projecting serotonergic neurons in the rostral ventromedial medulla is antinociceptive. Pain 114:285-294.

Burgess SE, Gardell LR, Ossipov MH, Malan TP Jr, Vanderah TW, Lai J, Porreca F (2002) Time-dependent descending facilitation from the rostral ventromedial medulla maintains, but does not initiate, neuropathic pain. J Neurosci 22:5129-5136.

Carlson JD, Maire JJ, Martenson ME, Heinricher MM (2007) Sensitization of pain-modulating neurons in the rostral ventromedial medulla after peripheral nerve injury. J Neurosci 27:13222-13231. 
Close LN, Cetas JS, Heinricher MM, Selden NR (2009) Purinergic receptor immunoreactivity in the rostral ventromedial medulla. Neuroscience 158:915-921.

Dogrul A, Ossipov MH, Porreca F (2009) Different mediation of descending pain facilitation and inhibition by spinal 5-HT-3 and 5-HT-7 receptors. Brain Res 1280:52-59.

Doucet E, Latrémolière A, Darmon M, Hamon M, Emerit MB (2007) Immunolabelling of the $5-\mathrm{HT} 3 \mathrm{~B}$ receptor subunit in the central and peripheral nervous systems in rodents. Eur J Neurosci 26:355-366.

Dubner R, Ren K (2004) Brainstem mechanisms of persistent pain following injury. J Orofacial Pain 18:299-305.

Fields HL, Heinricher MM (1989) Brainstem modulation of nociceptordriven withdrawal reflexes. Ann NY Acad Sci 563:34-44.

Fields HL, Basbaum AI, Heinricher MM (2006) Central nervous system mechanisms of pain modulation. In: Wall and Melzack's textbook of pain, Ed 5 (McMahon SB, Koltzenburg M, eds), pp 125-142. Philadelphia: Elsevier Churchill Livingstone.

Finnegan TF, Li DP, Chen SR, Pan HL (2004) Activation of mu-opioid receptors inhibits synaptic inputs to spinally projecting rostral ventromedial medulla neurons. J Pharmacol Exp Ther 309:476-483.

Gao K, Mason P (2000) Serotonergic raphe magnus cells that respond to noxious tail heat are not ON or OFF cells. J Neurophysiol 84:1719-1725.

Gao K, Chen DO, Genzen JR, Mason P (1998) Activation of serotonergic neurons in the raphe magnus is not necessary for morphine analgesia. J Neurosci 18:1860-1868.

Géranton SM, Fratto V, Tochiki KK, Hunt SP (2008) Descending serotonergic controls regulate inflammation-induced mechanical sensitivity and methyl-CpG-binding protein 2 phosphorylation in the rat superficial dorsal horn. Mol Pain 4:35.

Glaum SR, Proudfit HK, Anderson EG (1990) 5-HT3 receptors modulate spinal nociceptive reflexes. Brain Res 510:12-16.

Green GM, Scarth J, Dickenson A (2000) An excitatory role for 5-HT in spinal inflammatory nociceptive transmission; state-dependent actions via dorsal horn 5- $\mathrm{HT}_{3}$ receptors in the anaesthetized rat. Pain 89:81-88.

Guo W, Wei F, Zou S, Robbins MT, Sugiyo S, Ikeda T, Tu JC, Worley PF, Dubner R, Ren K (2004) Group I metabotropic glutamate receptor NMDA receptor coupling and signaling cascade mediate spinal dorsal horn NMDA receptor 2B tyrosine phosphorylation associated with inflammatory hyperalgesia. J Neurosci 24:9161-9173.

Guo W, Robbins MT, Wei F, Zou S, Dubner R, Ren K (2006) Supraspinal brain-derived neurotrophic factor signaling: a novel mechanism for pain facilitation. J Neurosci 26:126-137.

Hammond DL, Yaksh TL (1984) Antagonism of stimulation-produced antinociception by intrathecal administration of methysergide or phentolamine. Brain Res 298:329-337.

Hammond DL, Tyce GM, Yaksh TL (1985) Efflux of 5-hydroxytryptamine and noradrenaline into spinal cord superfusates during stimulation of the rat medulla. J Physiol 359:151-162.

Hamon M, Bourgoin S (1999) Serotonin and its receptors in pain control. In: Novel aspects of pain management: opioids and beyond (Sawynok J, Cowan A, eds), pp 203-228. New York: Wiley.

Hargreaves K, Dubner R, Brown F, Flores C, Joris J (1988) A new and sensitive method for measuring thermal nociception in cutaneous hyperalgesia. Pain 32:77-88.

Heinricher MM, Morgan MM, Tortorici V, Fields HL (1994) Disinhibition of off-cells and antinociception produced by an opioid action within the rostral ventromedial medulla. Neuroscience 63:279-288.

Hirata H, Takeshita S, Hu JW, Bereiter DA (2000) Cornea-responsive medullary dorsal horn neurons: modulation by local opioids and projections to thalamus and brain stem. J Neurophysiol 84:1050-1061.

Hökfelt T, Arvidsson U, Cullheim S, Millhorn D, Nicholas AP, Pieribone V, Seroogy K, Ulfhake B (2000) Multiple messengers in descending serotonin neurons: localization and functional implications. J Chem Neuroanat 18:75-86.

Hurley RW, Hammond DL (2001) Contribution of endogenous enkephalins to the enhanced analgesic effects of supraspinal mu opioid receptor agonists after inflammatory injury. J Neurosci 21:2536-2545.

Hurley RW, Banfor P, Hammond DL (2003) Spinal pharmacology of antinociception produced by microinjection of mu or delta opioid receptor agonists in the ventromedial medulla of the rat. Neuroscience 118:789-796.
Invernizzi RW (2007) Role of TPH-2 in brain function: news from behavioral and pharmacologic studies. J Neurosci Res 85:3030-3035.

Jeong CY, Choi JI, Yoon MH (2004) Roles of serotonin receptor subtypes for the antinociception of 5-HT in the spinal cord of rats. Eur J Pharmacol 502:205-211.

Johannessen JN, Watkins LR, Carlton SM, Mayer DJ (1982) Failure of spinal cord serotonin depletion to alter analgesia elicited from the periaqueductal gray. Brain Res 237:373-386.

Johansen JP, Fields HL (2004) Glutamatergic activation of anterior cingulate cortex produces an aversive teaching signal. Nat Neurosci 7:398-403.

Kalyuzhny AE, Wessendorf MW (1999) Serotonergic and GABAergic neurons in the medial rostral ventral medulla express kappa-opioid receptor immunoreactivity. Neurosci 90:229-234.

Kayser V, Elfassi IE, Aubel B, Melfort M, Julius D, Gingrich JA, Hamon M, Bourgoin S (2007) Mechanical, thermal and formalin-induced nociception is differentially altered in 5-HT1A-/-, 5-HT1B-/-, 5-HT2A-/-, 5-HT3A-/- and 5-HTT-/- knock-out male mice. Pain 130:235-248.

King VR, Michael GJ, Joshi RK, Priestley JV (1999) TrkA, trkB, and trkC messenger RNA expression by bulbospinal cells of the rat. Neuroscience 92:935-944.

Kovelowski CJ, Ossipov MH, Sun H, Lai J, Malan TP, Porreca F (2000) Supraspinal cholecystokinin may drive tonic descending facilitation mechanisms to maintain neuropathic pain in the rat. Pain 87:265-273.

Liu XY, Wu SX, Wang YY, Wang W, Zhou L, Li YQ (2005) Changes of 5-HT receptor subtype mRNAs in rat dorsal root ganglion by bee venominduced inflammatory pain. Neurosci Lett 375:42-46.

Lopez-Garcia JA (2006) Serotonergic modulation of spinal sensory circuits. Curr Top Med Chem 6:1987-1996.

Marinelli S, Vaughan CW, Schnell SA, Wessendorf MW, Christie MJ (2002) Rostral ventromedial medulla neurons that project to the spinal cord express multiple opioid receptor phenotypes. J Neurosci 22:10847-10855.

Millan MJ (2002) Descending control of pain. Prog Neurobiol 66:355-474.

Millan MJ, Girardon S, Bervoets K (1997) 8-OH-DPAT-induced spontaneous tail-flicks in the rat are facilitated by the selective serotonin (5-HT) $2 \mathrm{C}$ agonist, RO 60-0175: blockade of its actions by the novel 5-HT2C receptor antagonist SB 206,553. Neuropharmacology 36:743-745.

Oatway MA, Chen Y, Weaver LC (2004) The 5-HT3 receptor facilitates mechanical allodynia following spinal cord injury. Pain 110:259-268.

Pan ZZ, Williams JT, Osborne PB (1990) Opioid actions on single nucleus raphe magnus neurons from rat and guinea-pig in vitro. J Physiol 427:519-532.

Pan ZZ, Wessendorf MW, Williams JT (1993) Modulation by serotonin of the neurons in rat nucleus raphe magnus in vitro. Neuroscience 54:421-429.

Pan ZZ, Tershner SA, Fields HL (1997) Cellular mechanism for antianalgesic action of agonists of the kappa-opioid receptor. Nature 389:382-385.

Paul D, Yao D, Zhu P, Minor LD, Garcia MM (2001) 5-Hydroxytryptamine3 (5-HT3) receptors mediate spinal 5-HT antinociception: an antisense approach. J Pharmacol Exp Ther 298:674-678.

Paxinos G, Watson C (2005) The rat brain in stereotaxic coordinates, Ed 5. Elsevier.

Pertovaara A, Keski-Vakkuri U, Kalmari J, Wei H, Panula P (2001) Response properties of neurons in the rostroventromedial medulla of neuropathic rats: attempted modulation of responses by [1DMe] NPYF, a neuropeptide FF analogue. Neuroscience 105:457-468.

Porreca F, Burgess SE, Gardell LR, Vanderah TW, Malan TP Jr, Ossipov MH, Lappi DA, Lai J (2001) Inhibition of neuropathic pain by selective ablation of brainstem medullary cells expressing the mu-opioid receptor. J Neurosci 21:5281-5288.

Porreca F, Ossipov MH, Gebhart GF (2002) Chronic pain and medullary descending facilitation. Trends Neurosci 25:319-325.

Potrebic SB, Fields HL, Mason P (1994) Serotonin immunoreactivity is contained in one physiological cell class in the rat rostral ventromedial medulla. J Neurosci 14:1655-1665.

Rahman W, Suzuki R, Webber M, Hunt SP, Dickenson AH (2006) Depletion of endogenous spinal 5-HT attenuates the behavioural hypersensitivity to mechanical and cooling stimuli induced by spinal nerve ligation. Pain 123:264-274.

Ren K, Dubner R (2002) Descending modulation in persistent pain: an update. Pain 100:1-6.

Ren K, Dubner R (2007) Pain facilitation and activity-dependent plasticity 
in pain modulatory circuitry: role of BDNF-TrkB signaling and NMDA receptors. Mol Neurobiol 35:224-235.

Suzuki R, Morcuende S, Webber M, Hunt SP, Dickenson AH (2002) Superficial NK1-expressing neurons control spinal excitability through activation of descending pathways. Nat Neurosci 5:1319-1326.

Suzuki R, Rygh LJ, Dickenson AH (2004) Bad news from the brain: descending 5-HT pathways that control spinal pain processing. Trends Pharmacol Sci 25:613-617.

Svensson CI, Tran TK, Fitzsimmons B, Yaksh TL, Hua XY (2006) Descending serotonergic facilitation of spinal ERK activation and pain behavior. FEBS Lett 580:6629-6634.

Vasko MR, Pang IH, Vogt M (1984) Involvement of 5-hydroxytryptaminecontaining neurons in antinociception produced by injection of morphine into nucleus raphe magnus or onto spinal cord. Brain Res 306:341-348.

Vaughan CW, McGregor IS, Christie MJ (1999) Cannabinoid receptor activation inhibits GABAergic neurotransmission in rostral ventromedial medulla neurons in vitro. Br J Pharmacol 127:935-940.

Walther DJ, Peter JU, Bashammakh S, Hörtnagl H, Voits M, Fink H, Bader M (2003) Synthesis of serotonin by a second tryptophan hydroxylase isoform. Science 299:76.

Wang H, Wessendorf MW (1999) Mu- and delta-opioid receptor mRNAs are expressed in spinally projecting serotonergic and nonserotonergic neurons of the rostral ventromedial medulla. J Comp Neurol 404: 183-196.

Wei F, Dubner R, Ren K (1999a) Nucleus reticularis gigantocellularis and nucleus raphe magnus in the brain stem exert opposite effects on behavioral hyperalgesia and spinal Fos protein expression after peripheral inflammation. Pain 80:127-141.

Wei F, Zou S, Young A, Dubner R, Ren K (1999b) Effects of four herbal extracts on adjuvant-induced inflammation and hyperalgesia in rats. J Altern Complement Med 5:429-436.

Wei F, Wang GD, Kerchner GA, Kim SJ, Xu HM, Chen ZF, Zhuo M (2001)
Genetic enhancement of inflammatory pain by forebrain NR2B overexpression. Nat Neurosci 4:164-169.

Wei F, Xia XM, Tang J, Ao H, Ko S, Liauw J, Qiu CS, Zhuo M (2003) Calmodulin regulates synaptic plasticity in the anterior cingulate cortex and behavioral responses: a microelectroporation study in adult rodents. J Neurosci 23:8402-8409.

Winkler CW, Hermes SM, Chavkin CI, Drake CT, Morrison SF, Aicher SA (2006) Kappa opioid receptor (KOR) and GAD67 immunoreactivity are found in OFF and NEUTRAL cells in the rostral ventromedial medulla. J Neurophysiol 96:3465-3473.

Yaksh TL, Wilson PR (1979) Spinal serotonin terminal system mediates antinociception. J Pharmacol Exp Ther 208:446-453.

Zeitz KP, Guy N, Malmberg AB, Dirajlal S, Martin WJ, Sun L, Bonhaus DW, Stucky CL, Julius D, Basbaum AI (2002) The 5-HT3 subtype of serotonin receptor contributes to nociceptive processing via a novel subset of myelinated and unmyelinated nociceptors. J Neurosci 22:1010-1019.

Zhang L, Hammond DL (2010) Cellular basis for opioid potentiation in the rostral ventromedial medulla of rats with persistent inflammatory nociception. Pain 149:107-116.

Zhang X, Beaulieu JM, Sotnikova TD, Gainetdinov RR, Caron MG (2004) Tryptophan hydroxylase-2 controls brain serotonin synthesis. Science 305:217.

Zhao ZQ, Scott M, Chiechio S, Wang JS, Renner KJ, Gereau RW 4th, Johnson RL, Deneris ES, Chen ZF (2006) Lmxlb is required for maintenance of central serotonergic neurons and mice lacking central serotonergic system exhibit normal locomotor activity. J Neurosci 26:12781-12788.

Zhao ZQ, Chiechio S, Sun YG, Zhang KH, Zhao CS, Scott M, Johnson RL, Deneris ES, Renner KJ, Gereau RW 4th, Chen ZF (2007) Mice lacking central serotonergic neurons show enhanced inflammatory pain and an impaired analgesic response to antidepressant drugs. J Neurosci 27:6045-6053.

Zhuo M, Gebhart GF (1991) Spinal serotonin receptors mediate descending facilitation of a nociceptive reflex from the nuclei reticularis gigantocellularis and gigantocellularis pars alpha in the rat. Brain Res 550:35-48. 